Juliana Álvares ${ }^{1}$, Vânia Eloisa Araújo ${ }^{1}$

Jans Bastos Izidoro ${ }^{1}$, Carolina Zampirolli Dias ${ }^{1}$

Bruna de Oliveira Ascef ${ }^{1}$, Francisco de Assis Acurcio ${ }^{1}$

Karen Sarmento Costa ${ }^{2}$, Ediná Alves Costa ${ }^{3}$

Silvana Nair Leite ${ }^{4}$, Orlando Mário Soeiro ${ }^{2}$

Margô Gomes de Oliveira Karnikowski ${ }^{5}$

Ione Aquemi Guibu ${ }^{6}$, Augusto Afonso Guerra-Júnior ${ }^{1}$

\section{Are we really measuring access? Systematic review of access measures to medicines in Brazil}

\section{Estamos de fato medindo acesso? Revisão sistemática das medidas de acesso a medicamentos no Brasil}

Abstract | Introduction: Access to medicines (AAM) is a key component in enabling and measuring the performance of health systems.

Objectives: This study aims to determine, through a systematic review, the profile of studies and results of $A A M$ indicators in Brazil. Methods: A systematic review of observational studies was performed using articles found in US National Library of Medicine (PubMed), CENTRAL, LILACS and Essential Medicines and Health Products Information Portal (WHO) databases, including a manual search in the references provided by the studies included and the grey literature (Thesis Databases: Capes, Biblioteca Digital de Teses e Dissertações da USP, ProQuest Dissertação e Teses; Technical reports: WHO Medicines Strategy and research reports: PNAD, Ministério da Saúde, IBGE). Results: 29 studies were included. Among these, 19 evaluated access at municipal level, 9 to the public sector, 12 from the perspective of users, 22 discussed access to essential medicines, 14 evaluated more than one dimension of access and 24 presented indicators related to availability.

Among all dimensions of $A A M, 19$

different indicators were found. The indicator "physical availability" (PA) was used in 12 studies. Conclusion: These studies focus on availability and no studies so far have evaluated all dimensions of $A A M$. Patterns of access varied in the studies. This review stresses the need to develop proper guidelines to evaluate $A A M$, allowing for comparisons and evaluations of bealth systems over time.

Keywords | Access to Health Services; Brazil; Pharmaceutical Services.
Resumo| Introdução: Acesso a medicamentos é componente-chave na viabilização e mensuração do desempenho dos sistemas de saúde. Objetivos: Este estudo objetivou verificar, por meio de revisão sistemática, o perfil de estudos e os resultados dos indicadores de acesso a medicamentos no Brasil. Métodos: Revisão sistemática de estudos observacionais disponíveis nas bases de dados, US National Library of Medicine (PubMed), Cochrane Central Register of Controlled Trials (CENTRAL), Literatura Latino-Americana e do Caribe em Ciências da Saúde (LILACS), Centre of Reviens and Dissemination (CRD) e Essential Medicines and Health Products Information Portal (WHO), incluindo busca manual nas referências dos estudos incluídos e literatura cinzenta (Banco de Teses - Capes, Biblioteca Digital de Teses e Dissertações da USP, ProQuest Dissertação e Teses, relatórios técnicos do WHO Medicines Strategy e relatórios de pesquisa: PNAD, Ministério da Saúde, IBGE). Resultados: Foram incluídos 29 estudos sobre Acesso a Medicamentos. Destes, 19 estudos avaliaram acesso no nível municipal, 9 no setor público, 12 sob a perspectiva exclusiva dos usuários, 22 estudos avaliaram medicamentos essenciais, 14 avaliaram mais de uma dimensão do acesso e 24 estudos apresentaram indicadores relacionados à disponibilidade. Entre todas as dimensões do AAM, foram encontrados 19 indicadores distintos. O indicador "disponibilidade física" foi utilizado em 12 estudos. Conclusão: Os estudos tiveram foco na disponibilidade e nenhum estudo avaliou todas as dimensões do acesso. O nível de acesso foi variável entre os estudos. Esta avaliação levanta a necessidade de desenvolver uma diretriz para avaliar AAM, que fomente comparações e avaliações de desempenho dos sistemas de saúde ao longo do tempo.

Palavras-chave | Acesso aos Serviços de Saúde; Assistência Farmacêutica; Brasil.

\footnotetext{
${ }^{1}$ Universidade Federal de Minas Gerais, Belo Horizonte/MG, Brasil.

${ }^{2}$ Ministério da Saúde, Brasília/DF, Brasil.

${ }^{3}$ Universidade Federal da Bahia, Salvador/BA, Brasil.

${ }^{4}$ Universidade Federal de Santa Catarina, Florianópolis/SC, Brasil

${ }^{5}$ Universidade de Brasília, Brasília/DF, Brasil.

${ }^{6}$ Faculdade de Ciências Médicas da Santa Casa de São Paulo, São Paulo/SP, Brasil.
} 


\section{INTRODUÇÃO|}

Acesso a medicamentos é um dos componentes essenciais da atenção à saúde e componente-chave da realização progressiva do direito à saúde e do acesso universal aos cuidados de saúde ${ }^{1-4}$. No contexto do Sistema Único de Saúde (SUS) é de suma importância a avaliação do acesso dos usuários às tecnologias de saúde (incluindo os medicamentos), bem como a qualidade desse acesso. Este aspecto se torna ainda mais relevante porque a garantia à saúde está inscrita na Constituição Federal de 1988, tendo o Estado dever de assegurar a saúde de forma universal, integral e equânime ${ }^{5}$. A Lei Orgânica da Saúde explicita o acesso a medicamentos, ao estabelecer que cabe ao SUS a execução de ações de assistência integral, inclusive farmacêutica ${ }^{6}$.

A Organização Mundial de Saúde define acesso a medicamentos como a disponibilidade e a capacidade financeira para sua obtenção de forma equitativa ${ }^{7}$. Entretanto, este conceito não leva em consideração a complexidade da efetivação do acesso a medicamentos, que envolve uma rede de atores públicos e privados e que desempenham diferentes papéis em função do contexto econômico, político e social. Dentro deste contexto encontra-se o usuário, sujeito com crenças individuais, conhecimento prévio e preferências em relação ao cuidado com a saúde. Assim, a simples disponibilidade do serviço não configura o acesso, uma vez que o acesso seria um fator mediador entre a capacidade de produzir e oferecer produtos ou serviços e o consumo real ${ }^{8}$.

Penchansky e Thomas" abordam o acesso de forma multidimensional, defindo-o como o "grau de ajuste entre os clientes e o sistema" o qual estaria representado nas seguintes dimensões: Disponibilidade, Acessibilidade geográfica, Acomodação, Capacidade aquisitiva e Aceitabilidade. Assim, compreender o acesso e suas dimensões é fundamental para que ocorra uma mensuração real da relação entre a necessidade e a oferta de medicamentos. Por isso, a medida do acesso é tão importante, uma vez que indica a resolubilidade e a qualidade de um sistema de saúde, além de contribuir para a elaboração de estratégias de construção, ampliação, intervenção e reorientação das políticas de medicamentos e de assistência farmacêutica ${ }^{10}$.

Outro ponto que reforça a necessidade deste monitoramento é o fato de que os dados da Pesquisa Nacional por Amostra de Domicílios (PNAD) 2012 e da Pesquisa de
Orçamentos Familiares (POF) 2008-2009 indicam que os programas de assistência farmacêutica do SUS são a única forma de acesso a medicamentos para grande parcela da população brasileira, principalmente aquela de menor renda ${ }^{11}$. Ademais, é observada uma ampliação expressiva do recurso federal para garantir o acesso a medicamentos à população brasileira no período entre 2003 e 2014, passando o orçamento de 1,9 bilhão para 12,4 bilhões, com investimento superior a 80 bilhões de reais ${ }^{12}$.

O SUS avançou consideravelmente nos últimos anos na organização de programas com a finalidade de garantir o acesso da população a medicamentos. Dentre as estratégias governamentais para garantia do acesso estão os programas mundialmente reconhecidos tais como o programa de medicamentos genéricos, o programa de DTS/AIDS ${ }^{13}$, o programa nacional de imunizações ${ }^{14}$. Destacam-se ainda a regulamentação dos blocos de financiamento da Assistência Farmacêutica (AF), a instituição e revisão periódica da Relação Nacional de Medicamentos Essenciais (RENAME) e mais recentemente, os programas Farmácia Popular do Brasil, Aqui Tem Farmácia Popular/Saúde Não Tem Preço (ATFP/SNTP) e o Programa Nacional de Qualificação da Assistência Farmacêutica (QUALIFAR-SUS).

Dados do Ministério da Saúde ${ }^{15}$ revelam que o acesso a medicamentos para hipertensão, diabetes e asma aumentou até 343\% nos últimos anos com o programa "Aqui Tem Farmácia Popular/Saúde Não Tem preço" e que uma das consequências disso foi a estabilização do número de internações por complicações decorrentes do diabetes desde 2010. Entretanto, vários autores ${ }^{16-19}$ identificaram disparidades e deficiências no acesso a medicamentos pela população.

O cenário é de incerteza acerca dos resultados dos indicadores de acesso a medicamentos no Brasil, mesmo com incrementos no financiamento das políticas públicas de medicamentos. Por outro lado, não existe, até o momento, uma definição operacional de acesso a medicamentos, nem um modelo acabado de avaliação que permita monitorar o acesso em um determinado país ao longo do tempo ou a comparação do desempenho entre países ${ }^{20}$.

Portanto, o objetivo deste estudo foi investigar, por meio de revisão sistemática da literatura, quais indicadores estão sendo utilizados para medir acesso a medicamentos no Brasil e seus respectivos resultados. 


\section{MÉTODOS|}

Foi conduzida uma revisão sistemática de estudos observacionais que apresentaram resultados quantitativos de medidas de acesso a medicamentos no Brasil. O artigo foi preparado usando o Preferred Reporting Items for Systematic Reviews and Meta-Analysis (PRISMA).

Foi realizada uma busca eletrônica de artigos relevantes publicados até fevereiro de 2015, nas bases de dados US National Library of Medicine (PubMed), Lilacs, Centre of Reviews and Dissemination (CRD), Cochrane Central Register of Controlled Trials (CENTRAL) e Essential Medicines and Health Products Information Portal (WHO). Para cada base de dados foram elaboradas estratégias de busca utilizando termos indexados e sinônimos (Tabela 1). Também foi realizada a busca manual nas referências dos estudos incluídos e na literatura cinzenta nas bases do Banco de Teses do portal CAPES, Biblioteca Digital de Teses e Dissertações da Universidade de São Paulo (USP), ProQuest Dissertação e Teses, relatórios técnicos do WHO Medicines Strategy e relatórios de pesquisa: PNAD, Ministério da Saúde, Instituto Brasileiro de Geografia e Estatística (IBGE).

O processo de seleção dos estudos foi realizado em três fases, por dois revisores independentes em duplicata e incluiu a análise de títulos, resumos e textos na íntegra. Divergências foram analisadas por um terceiro revisor.

A coleta de dados foi realizada por três pesquisadores em um formulário eletrônico padronizado que compilou informações de cada estudo sobre: classificação da dimensão do acesso avaliada, sistematizada por um modelo conceitual, tomandose por base Penchansky e Thomas ${ }^{9}$ (Disponibilidade,

Tabela 1 - Estratégias de busca utilizadas nas bases de dados selecionadas

\begin{tabular}{|c|c|c|}
\hline Base de Dados & Estratégia de busca (realizada em 25/02/2015) & $\mathbf{N}$ \\
\hline CRD & (Brazil or brazilian [Any field]) AND (drugs or medicines [Any field]) & 56 \\
\hline WHO & $\begin{array}{l}\text { Text Words "Medicines" OR "Drugs" AND Text words: "Access" OR Subject: "Financing” OR } \\
\text { "Good Governance for Medicines" OR "Pricing" OR "Primary Health Care" OR "Selection" OR } \\
\text { "Supply Management" OR "Procurement" OR "Needs assessment and planing" AND Country: } \\
\text { "Brazil”. }\end{array}$ & 62 \\
\hline PubMed & $\begin{array}{c}\text { ((((((“Pharmaceutical Preparations”[Mesh]) OR Pharmaceutical Preparations[Text Word]) } \\
\text { OR Drugs[Text Word]) OR Medicines[Text Word])) AND (((((((((access[Text Word]) OR } \\
\text { "Health Services Accessibility”[Mesh]) OR Accessibility[Text Word]) OR Affordability[Text } \\
\text { Word]) OR “Patient Acceptance of Health Care"[Mesh]) OR Acceptability[Text Word]) OR } \\
\text { (“supply and distribution” [Subheading]))) OR “Quality Indicators, Health Care”[Mesh])) AND } \\
\text { (((“Brazil”[Mesh]) OR Brazil[Text Word]) OR brazilian[Text Word]) }\end{array}$ & 440 \\
\hline LILACS & $\begin{array}{c}\text { (((Preparações farmacêuticas) OR (Preparaciones Farmacéuticas) OR (Pharmaceutical } \\
\text { Preparations) OR (VS2.002.001) OR (Drugs OR drogas OR fármacos OR medicamentos } \\
\text { OR medicine\$)) AND (access OR acess\$ OR acesso OR acceso OR (Health Services } \\
\text { Accessibility) OR (Accesibilidad a los Servicios de Salud) OR (Acesso aos Serviços de } \\
\text { Saúde) OR (N04.590.374.350) OR (N05.300.430) OR (SP1.001.032) OR (Health Care } \\
\text { Quality, Access, and Evaluation) OR (Calidad, Acceso y Evaluación de la Atención de Salud) } \\
\text { OR (Qualidade, Acesso e Avaliação da Assistência à Saúde) OR (N05)) AND (Brazil OR } \\
\text { Brasil OR Brazil\$ OR Brasil\$) }\end{array}$ & 620 \\
\hline CENTRAL & $\begin{array}{l}\text { MeSH descriptor: [Pharmaceutical Preparations] OR drug:ti,ab,kw OR medicine:ti,ab,kw } \\
\text { (Word variations have been searched) OR Pharmaceutical Preparations:ti,ab,kw (Word } \\
\text { variations have been searched) AND MeSH descriptor: [Health Services Accessibility] OR } \\
\text { MeSH descriptor: [Health Care Quality, Access, and Evaluation] OR access:ti,ab,kw OR } \\
\text { Any MeSH descriptor with qualifier(s): [Supply \& distribution - SD] OR Accessibility:ti,ab,kw } \\
\text { (Word variations have been searched) OR Affordability:ti,ab,kw (Word variations have been } \\
\text { searched) OR MeSH descriptor: [Patient Acceptance of Health Care] OR Acceptability:ti,ab,kw } \\
\text { (Word variations have been searched) OR MeSH descriptor: [Quality Indicators, Health Care] } \\
\text { AND MeSH descriptor: [Brazil] OR brazil:ti,ab,kw (Word variations have been searched) OR } \\
\text { brazilian:ti,ab,kw (Word variations have been searched) }\end{array}$ & 370 \\
\hline
\end{tabular}


Acessibilidade geográfica, Acomodação, Capacidade aquisitiva e Aceitabilidade); Abrangência da amostra do estudo no Brasil (nacional, estadual, municipal); Ano de publicação do estudo e realização da pesquisa; Região onde foi realizada a pesquisa (Norte, Sul, Centro-Oeste, Sudeste e Nordeste); fonte de informação (usuários, gestores, profissionais de saúde, observação in loco); tipo de medicamentos avaliados e setor de avaliação (privado, público, filantrópico).

O processo de avaliação da qualidade metodológica dos estudos também foi realizado de forma independente por dois revisores, com a participação de um terceiro revisor, quando não houve consenso. Foi utilizada a ferramenta Quality Assessment Tool for Observational Cohort and Cross-Sectional Studies, do National Institute of Health - EUA (NIH).

\section{RESULTADOS |}

Após pesquisa nas bases de dados foram identificadas 1548 publicações para seleção dos estudos, sendo incluídos
29 estudos que relataram acesso a medicamentos como desfecho primário ${ }^{21-49}$. A figura 1 ilustra o fluxo de avaliação da inclusão e exclusão dos estudos.

Dentre os 29 estudos incluídos, foi encontrada somente uma coorte sobre acesso ${ }^{22}$ e todos os outros estudos foram de delineamento transversal. No total, seis estudos (21\%) avaliaram o acesso exclusivamente no setor privado, nove (31\%) exclusivamente no setor público, 12 (41\%) no público e privado concomitantemente, um estudo (3\%) avaliou o acesso a medicamentos em instituição filantrópica (terceiro setor) e um $(3 \%)$ avaliou nos setores privado, público e terceiro setor. Considerando a abrangência dos estudos, sete (24\%) avaliaram o acesso no nível nacional, três (10\%) no nível estadual e 19 $(66 \%)$ no nível municipal. Avaliando a fonte de informação do acesso nos estudos incluídos, observou-se que em 12 estudos (41\%) a informação foi obtida pelo pesquisador por meio de observação in loco, em 12 (41\%) pelo usuário e um estudo pelo gestor. Nos outros quatro estudos a fonte de informação foi mista: pesquisador e usuário (dois estudos); pesquisador e profissional (um estudo); pesquisador, usuário e profissional (um estudo) (Quadro 1).

Figura 1 - Diagrama do fluxo de avaliação da inclusão/ exclusão dos estudos da revisão sistemática

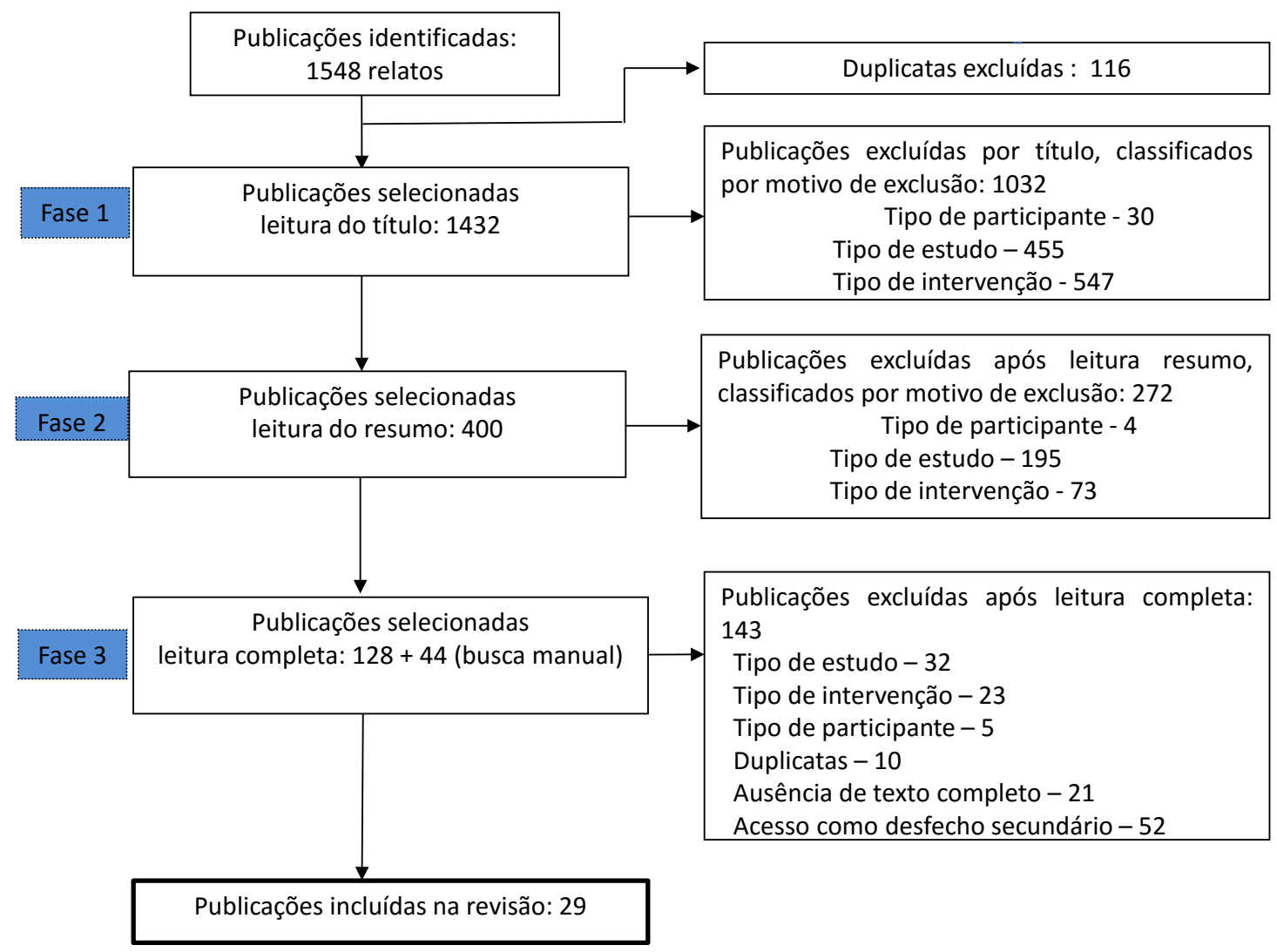

Rev. Bras. Pesq. Saúde, Vitória, 17(4): 138-155, out-dez, 2015 | 141 


\begin{tabular}{|c|c|c|c|c|c|c|}
\hline 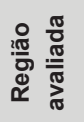 & \multicolumn{3}{|c|}{$\begin{array}{l}\frac{\mathscr{D}}{\omega} \\
\frac{\mathbb{0}}{0} \\
\omega\end{array}$} & \multicolumn{2}{|r|}{$\overline{\bar{\Xi}}$} & $\begin{array}{l}\frac{1}{\omega} \\
\frac{0}{0} \\
心\end{array}$ \\
\hline 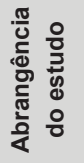 & \multicolumn{3}{|c|}{ 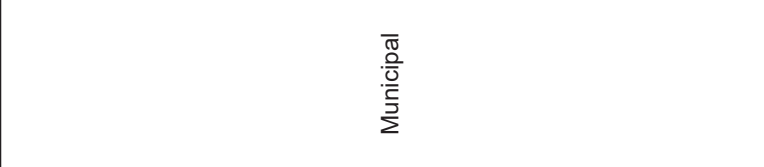 } & \multicolumn{2}{|r|}{ 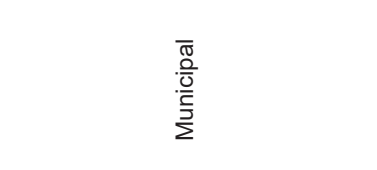 } & 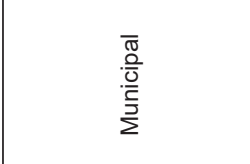 \\
\hline 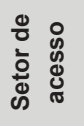 & \multicolumn{3}{|c|}{ 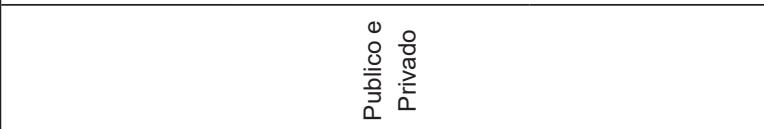 } & \multicolumn{2}{|r|}{$\frac{.0}{0}$} & 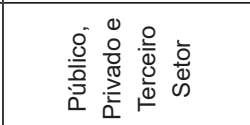 \\
\hline 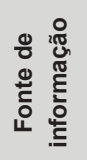 & \multicolumn{3}{|c|}{ 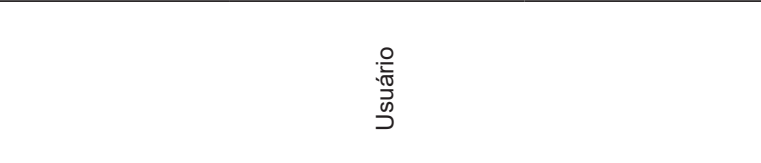 } & \multicolumn{2}{|r|}{ 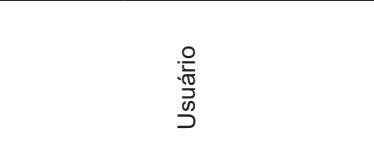 } & 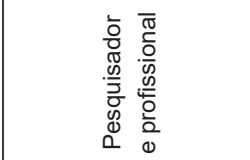 \\
\hline 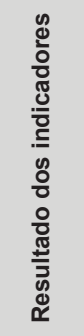 & 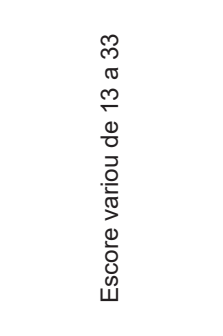 & 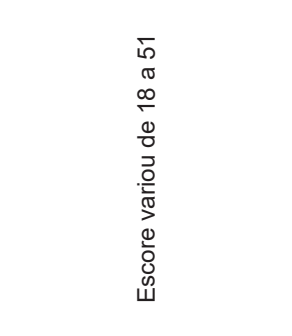 & 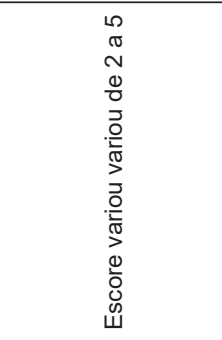 & 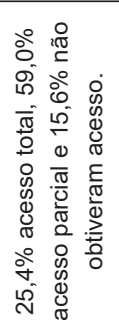 & 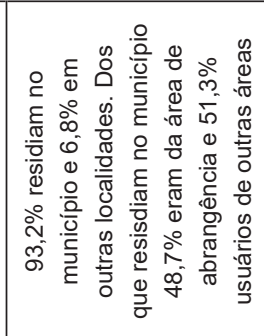 & 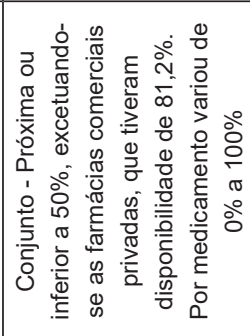 \\
\hline 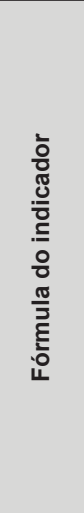 & 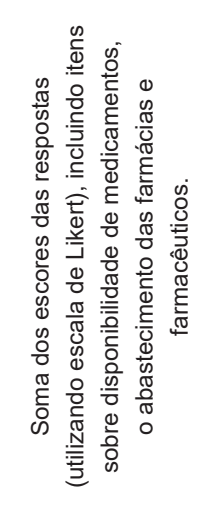 & 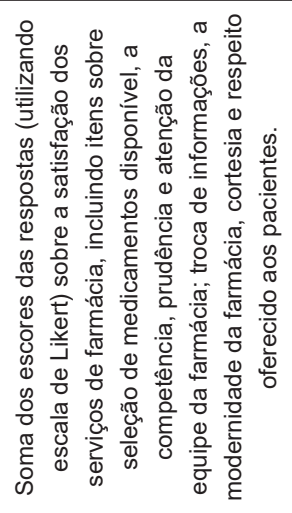 & 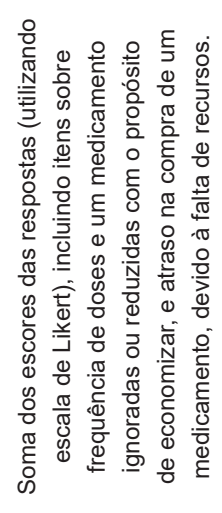 & 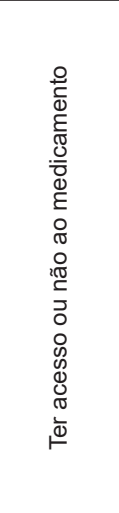 & 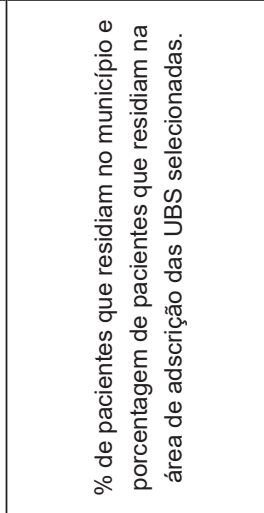 & 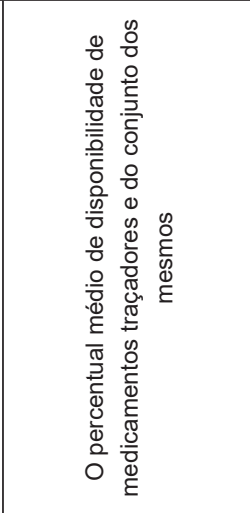 \\
\hline 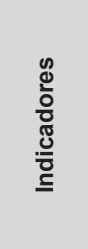 & 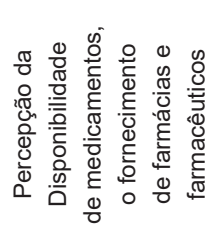 & 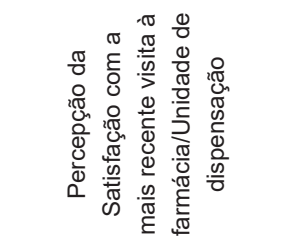 & 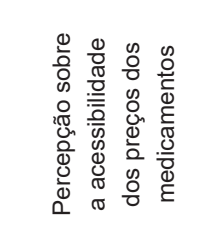 & 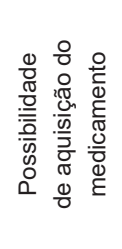 & 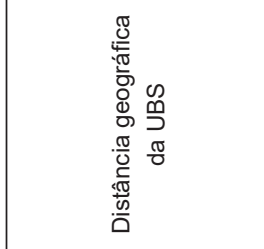 & 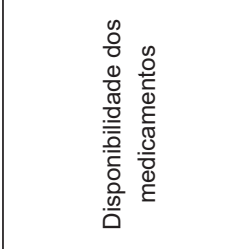 \\
\hline 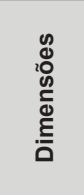 & 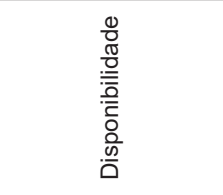 & $\begin{array}{l}\frac{0}{0} \\
\frac{\pi}{0} \\
\frac{0}{\overline{0}} \\
\frac{\pi}{\pi} \\
\frac{\pi}{\mathbb{1}} \\
\frac{0}{4}\end{array}$ & 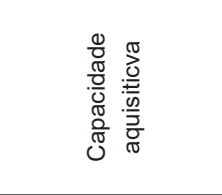 & 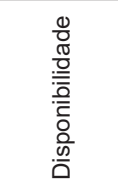 & 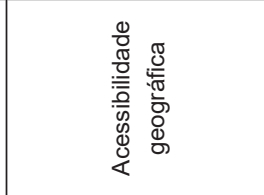 & 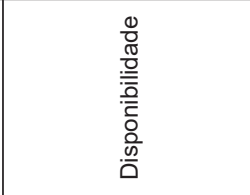 \\
\hline 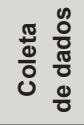 & & $\stackrel{\circ}{\stackrel{\circ}{\circ}}$ & & & $\stackrel{\widehat{ఠ}}{\circ}$ & চ্̀ \\
\hline 율 & & 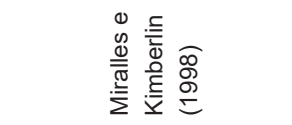 & & & 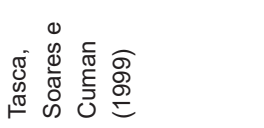 & 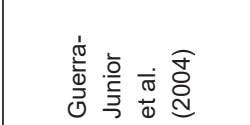 \\
\hline
\end{tabular}




\begin{tabular}{|c|c|c|c|c|c|c|c|c|c|c|}
\hline 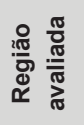 & \multicolumn{7}{|c|}{$\begin{array}{l}\frac{0}{\Phi} \\
\frac{0}{0} \\
\omega\end{array}$} & \multicolumn{2}{|l|}{$\begin{array}{l}\overline{\bar{w}} \\
\overline{\mathbb{D}} \\
\bar{\Phi}\end{array}$} & $\overline{\bar{D}}$ \\
\hline 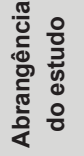 & \multicolumn{7}{|c|}{ 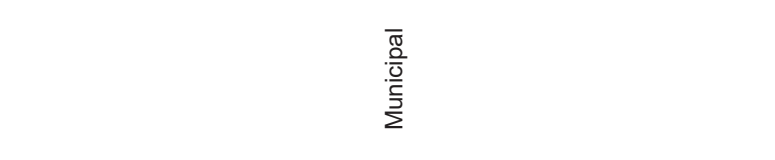 } & \multicolumn{2}{|l|}{$\begin{array}{l}\bar{\pi} \\
\bar{\sigma} \\
\frac{0}{0} \\
\frac{\pi}{z}\end{array}$} & 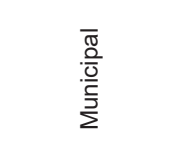 \\
\hline 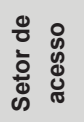 & \multicolumn{7}{|c|}{$\frac{8}{\frac{8}{0}}$} & \multicolumn{2}{|l|}{ 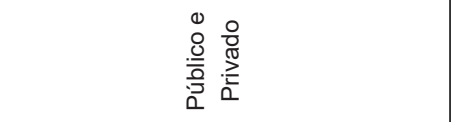 } & 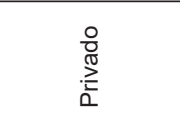 \\
\hline 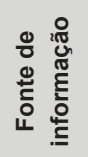 & \multicolumn{7}{|c|}{ 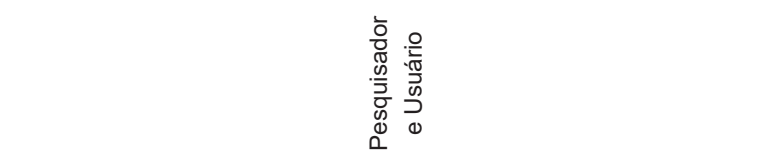 } & \multicolumn{2}{|l|}{ 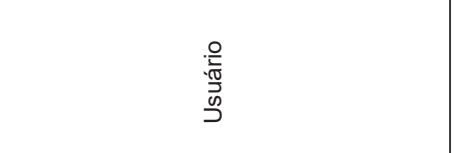 } & 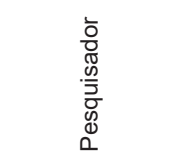 \\
\hline 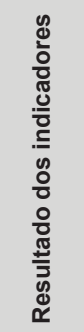 & $\begin{array}{l}\stackrel{0}{0} \\
0 \\
0 \\
0\end{array}$ & 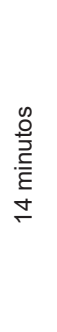 & هें & ญें & ஓे & 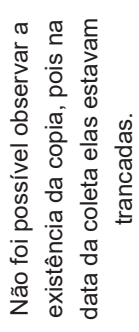 & 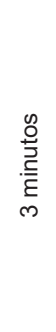 & 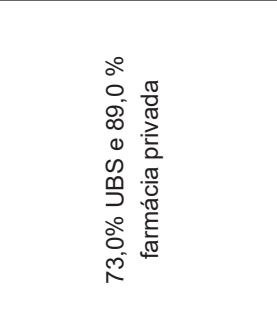 & 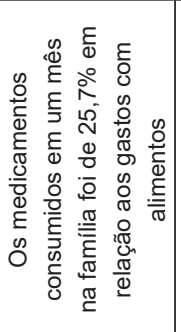 & 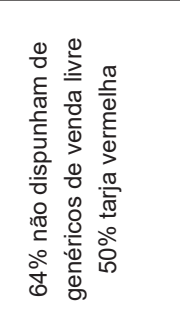 \\
\hline 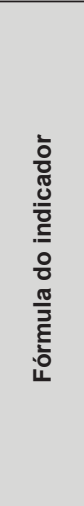 & 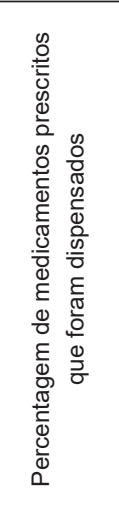 & 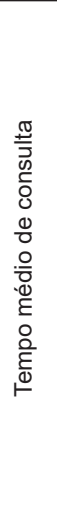 & 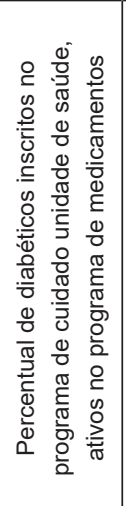 & 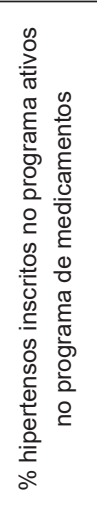 & 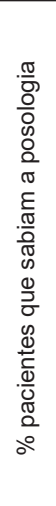 & 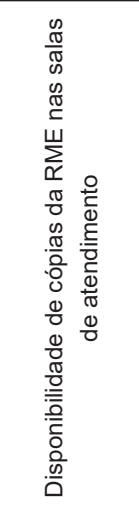 & 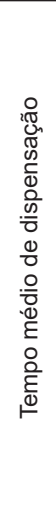 & 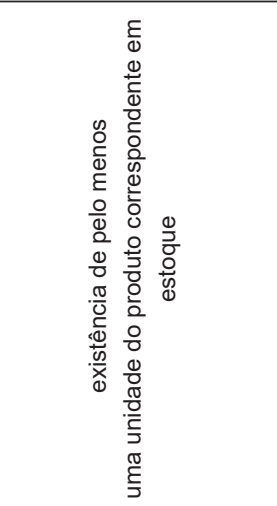 & 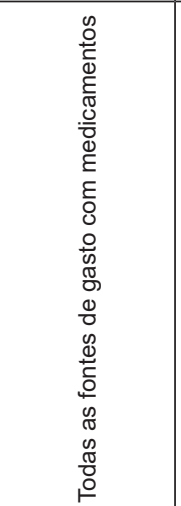 & 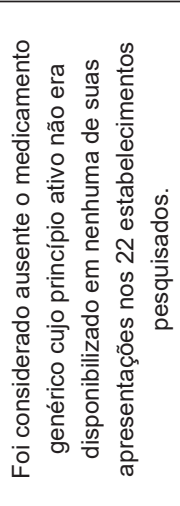 \\
\hline 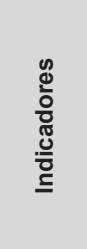 & 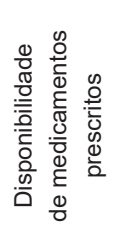 & \multicolumn{6}{|c|}{ 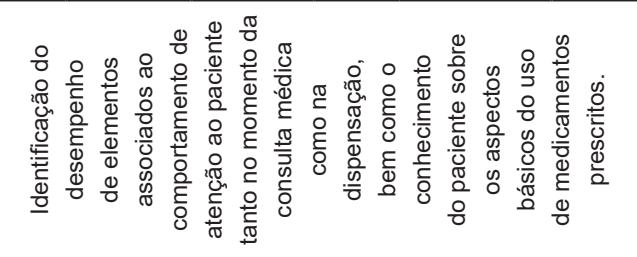 } & 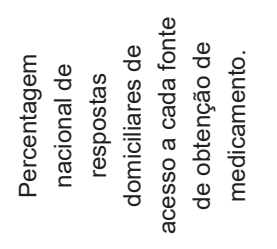 & 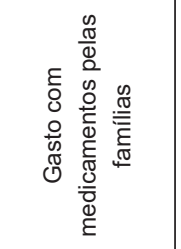 & 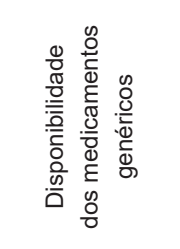 \\
\hline 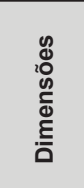 & 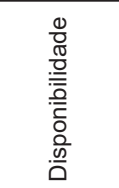 & \multicolumn{6}{|c|}{ 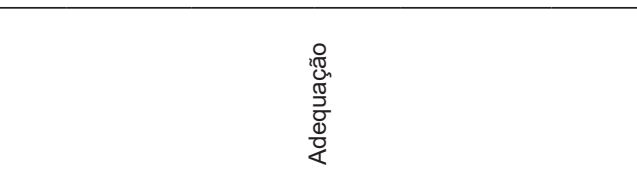 } & 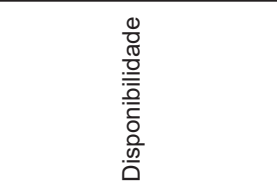 & 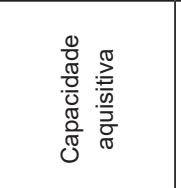 & 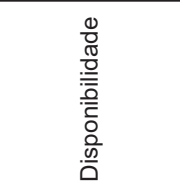 \\
\hline $\begin{array}{l}\frac{n}{0} \\
\frac{0}{0} \\
\frac{0}{0} \\
0 \\
0\end{array}$ & \multicolumn{7}{|c|}{ ণ్ } & \multicolumn{2}{|l|}{ ণ্ } & ڤ్ \\
\hline 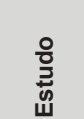 & \multicolumn{7}{|c|}{ 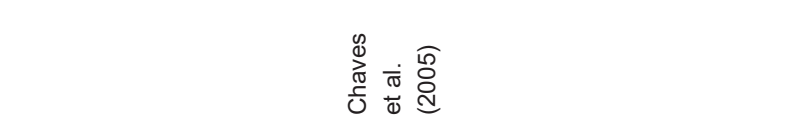 } & \multicolumn{2}{|l|}{ 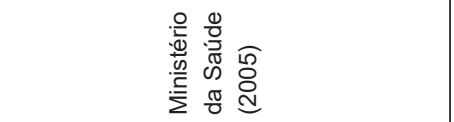 } & 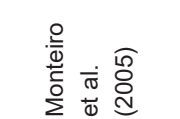 \\
\hline
\end{tabular}

Rev. Bras. Pesq. Saúde, Vitória, 17(4): 138-155, out-dez, 2015 | 143 


\begin{tabular}{|c|c|c|c|c|c|}
\hline 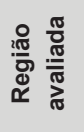 & \multicolumn{3}{|c|}{$\overline{\bar{S}}$} & 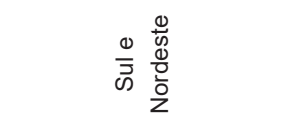 & $\overline{\bar{B}}$ \\
\hline 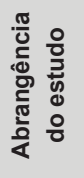 & \multicolumn{3}{|c|}{ 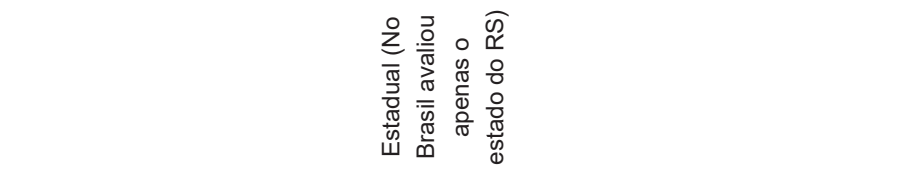 } & 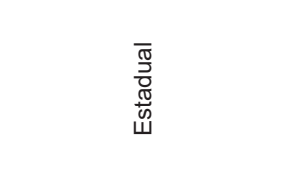 & 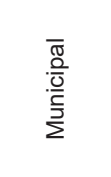 \\
\hline 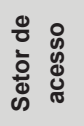 & \multicolumn{3}{|c|}{ 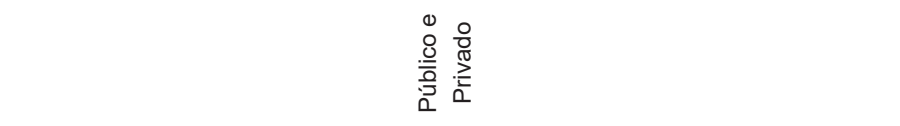 } & $\frac{8}{\frac{8}{\overline{3}}}$ & $\frac{8}{\overline{0}}$ \\
\hline 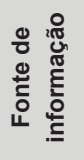 & \multicolumn{3}{|c|}{ 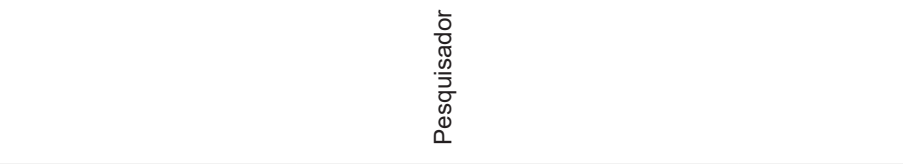 } & 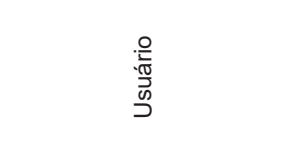 & 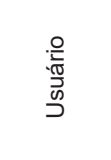 \\
\hline 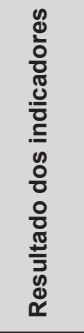 & 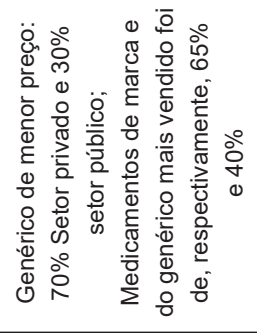 & 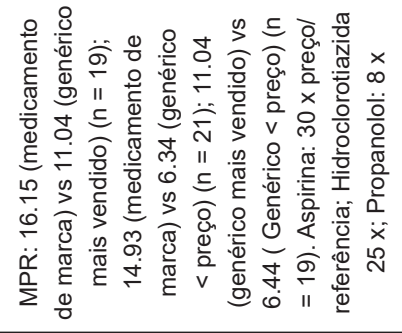 & 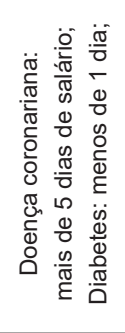 & 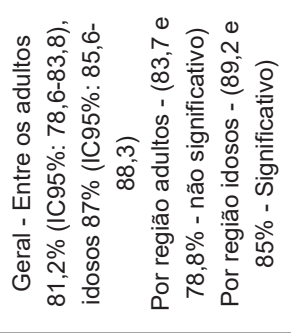 & 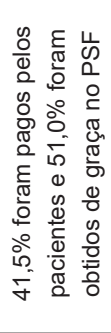 \\
\hline 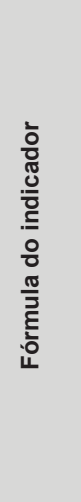 & 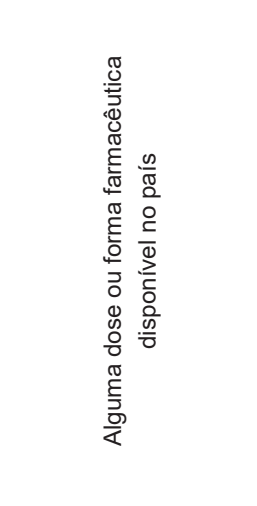 & 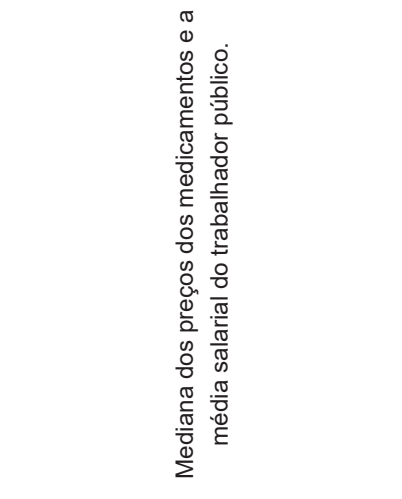 & 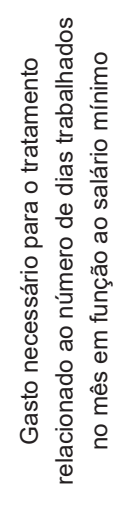 & 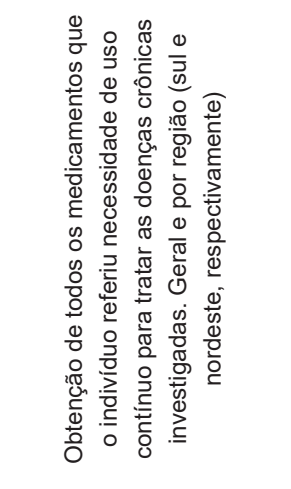 & 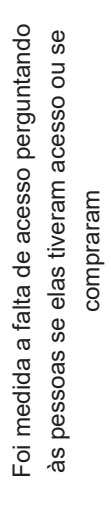 \\
\hline 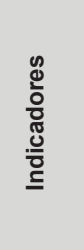 & 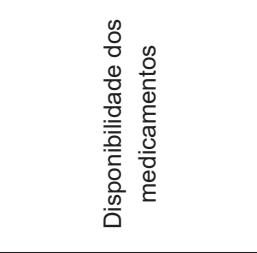 & 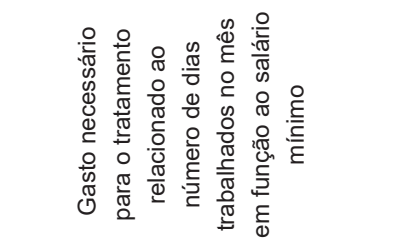 & 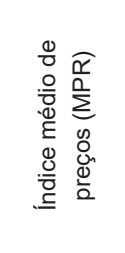 & 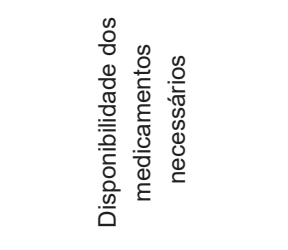 & 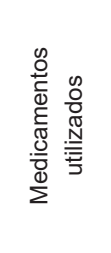 \\
\hline 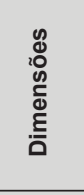 & 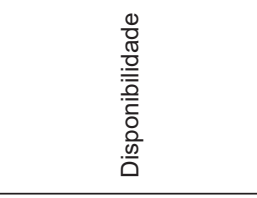 & \multicolumn{2}{|l|}{ 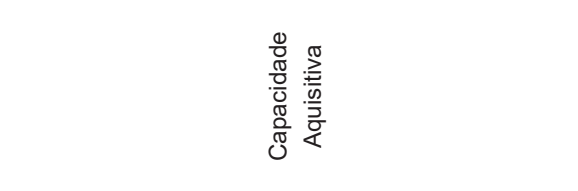 } & 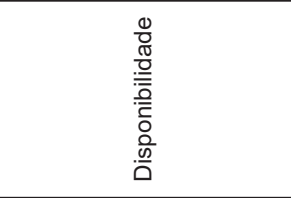 & 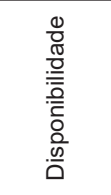 \\
\hline 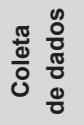 & \multicolumn{3}{|c|}{ 苂 } & 苂 & ֻ \\
\hline 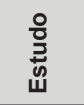 & \multicolumn{3}{|c|}{ 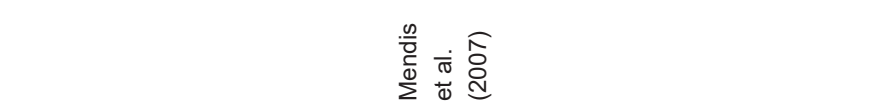 } & 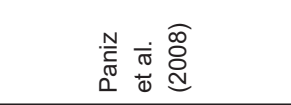 & 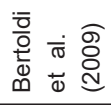 \\
\hline
\end{tabular}




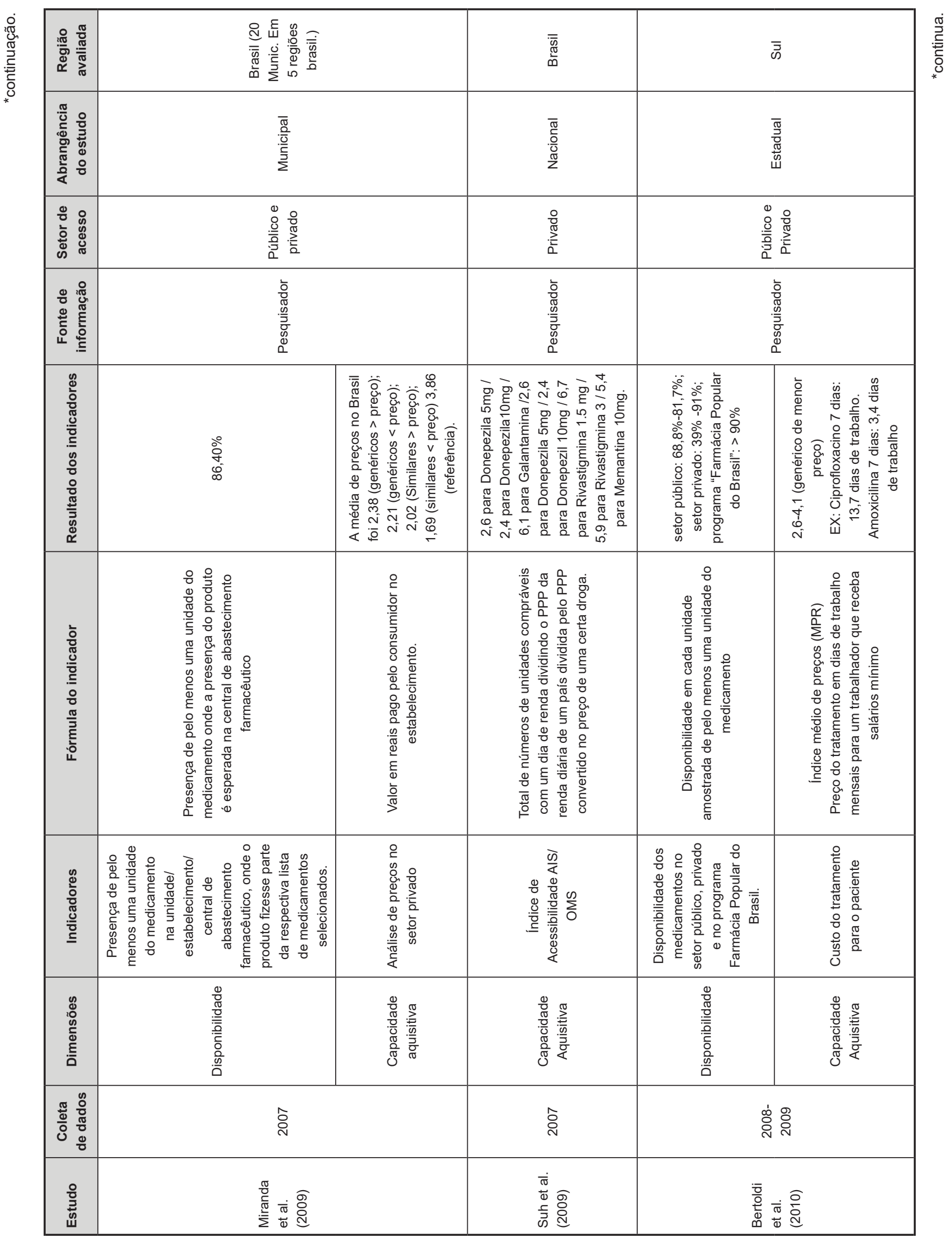

Rev. Bras. Pesq. Saúde, Vitória, 17(4): 138-155, out-dez, 2015 | 145 


\begin{tabular}{|c|c|c|c|c|c|c|}
\hline 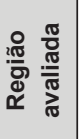 & & $\begin{array}{l}\frac{1}{0} \\
\frac{0}{0} \\
\omega \\
\omega\end{array}$ & 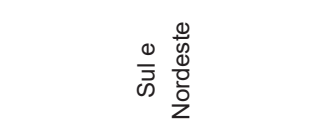 & \multicolumn{2}{|l|}{ 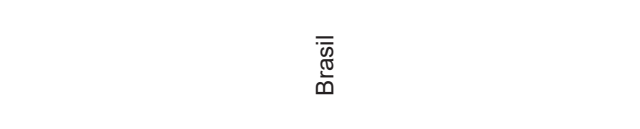 } & $\begin{array}{l}\frac{9}{\tilde{D}} \\
\frac{0}{0} \\
\omega\end{array}$ \\
\hline 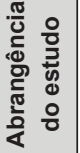 & \multicolumn{2}{|r|}{ 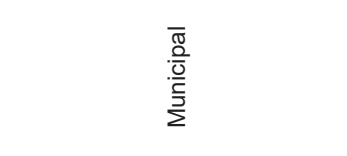 } & 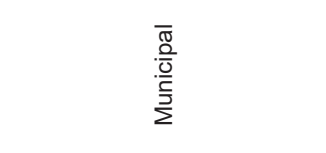 & \multicolumn{2}{|l|}{ 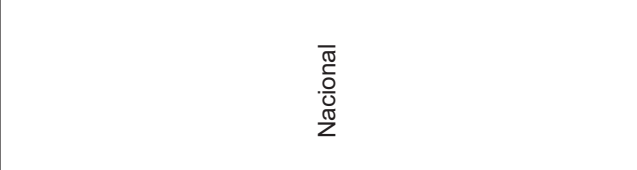 } & 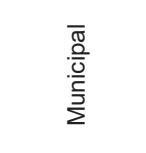 \\
\hline 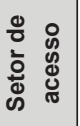 & \multicolumn{2}{|r|}{ 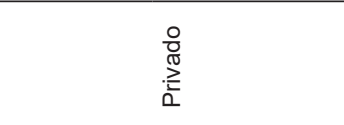 } & $\frac{.0}{\frac{0}{\overline{3}}}$ & \multicolumn{2}{|l|}{ 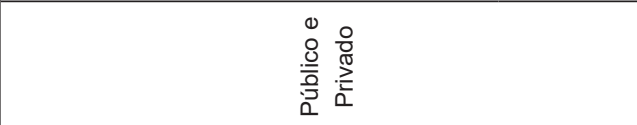 } & 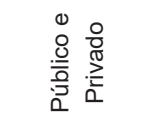 \\
\hline 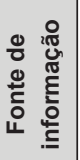 & \multicolumn{2}{|c|}{ 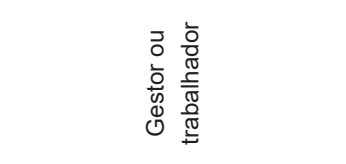 } & 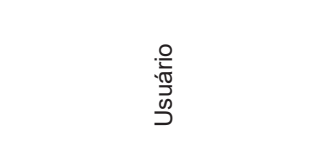 & \multicolumn{2}{|l|}{ 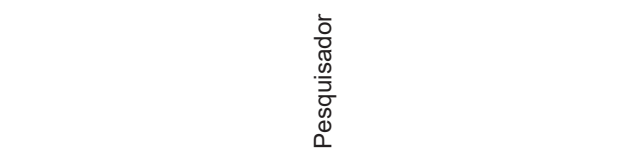 } & 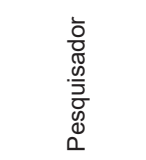 \\
\hline 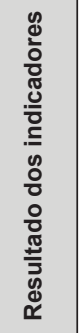 & 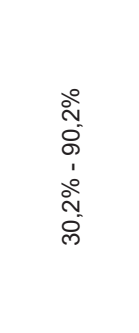 & 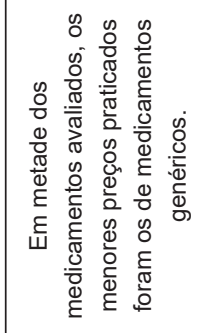 & 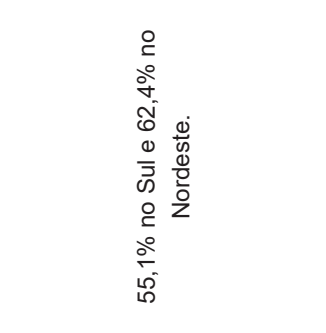 & 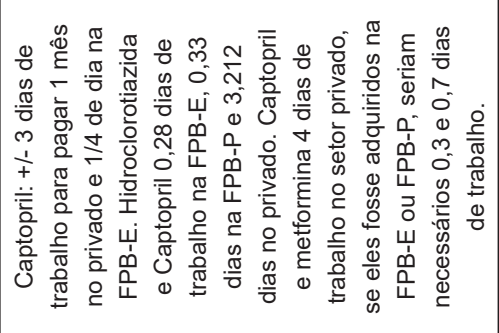 & $\frac{\circ}{\stackrel{0}{0}}$ & 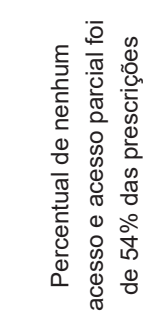 \\
\hline 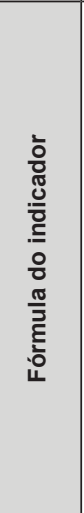 & 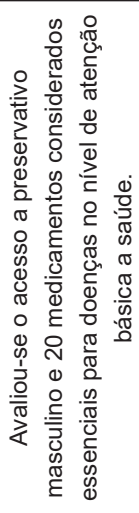 & 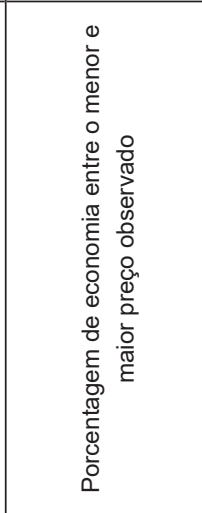 & 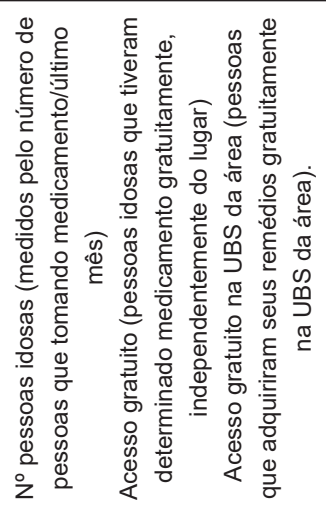 & 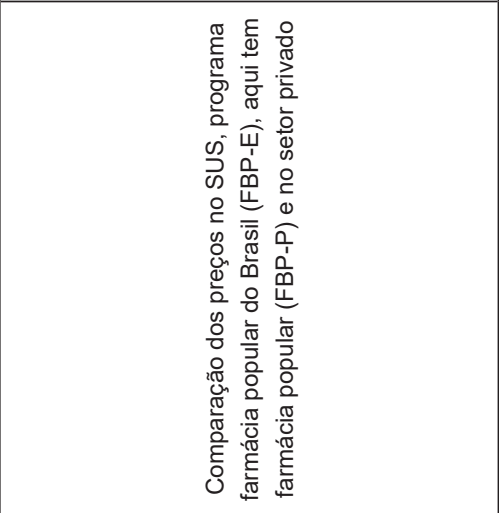 & 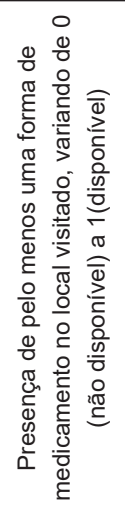 & 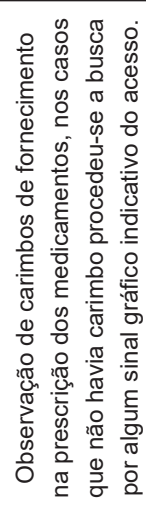 \\
\hline 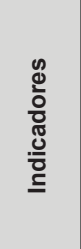 & 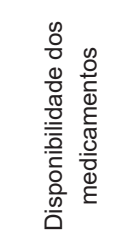 & 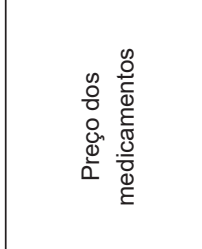 & 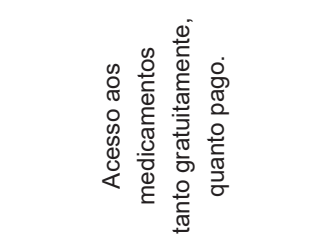 & 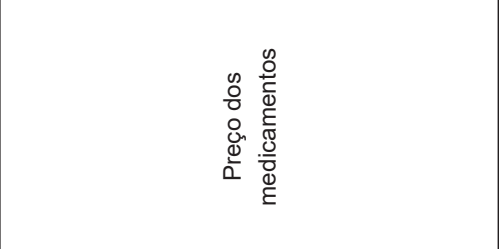 & 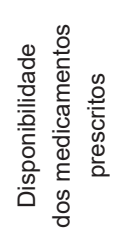 & 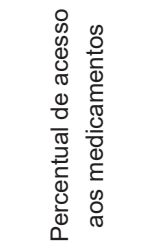 \\
\hline 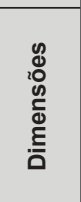 & 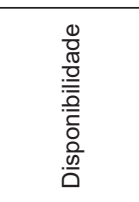 & 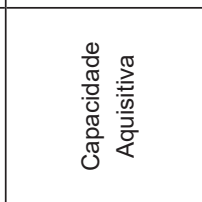 & 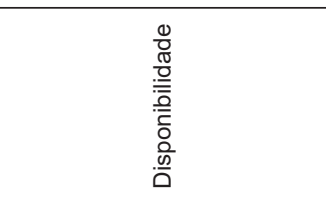 & 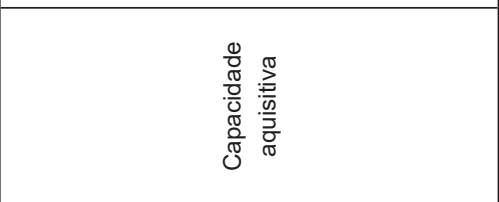 & 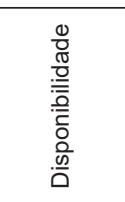 & 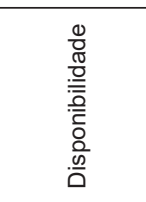 \\
\hline 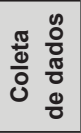 & \multicolumn{2}{|r|}{ ڤ్ } & ֻั & \multicolumn{2}{|l|}{ ڤ్̀ } & ֻัم \\
\hline $\begin{array}{l}\text { 을 } \\
\text { 离 } \\
\text { W }\end{array}$ & \multicolumn{2}{|c|}{ 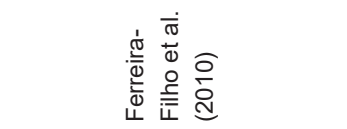 } & 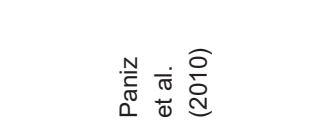 & \multicolumn{2}{|l|}{ 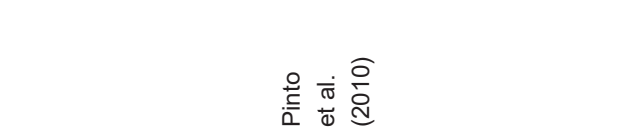 } & 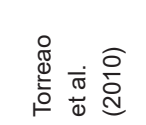 \\
\hline
\end{tabular}




\begin{tabular}{|c|c|c|c|c|c|c|c|c|}
\hline 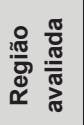 & \multicolumn{3}{|c|}{$\begin{array}{l}\frac{\Phi}{w} \\
\frac{0}{0} \\
心\end{array}$} & \multicolumn{2}{|l|}{$\overline{\bar{D}}$} & $\overline{\bar{B}}$ & $\overline{\bar{S}}$ & $\overline{\bar{S}}$ \\
\hline 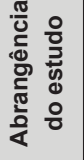 & \multicolumn{3}{|c|}{ 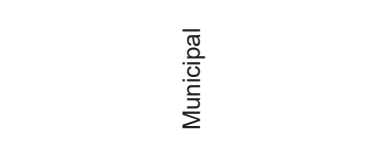 } & \multicolumn{2}{|l|}{ 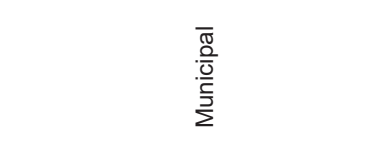 } & 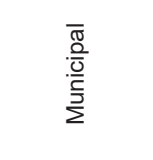 & 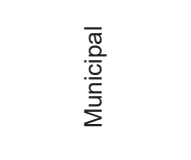 & 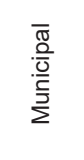 \\
\hline 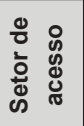 & \multicolumn{3}{|c|}{$\frac{8}{\frac{8}{0}}$} & \multicolumn{2}{|l|}{ 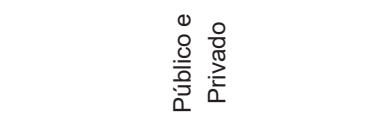 } & 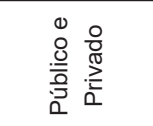 & 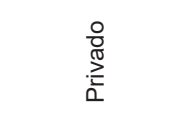 & $\frac{8}{\frac{8}{0}}$ \\
\hline 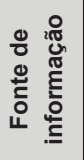 & \multicolumn{3}{|c|}{ 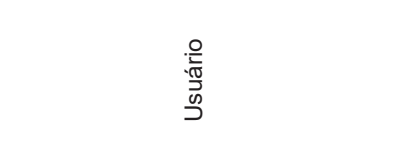 } & \multicolumn{2}{|l|}{ 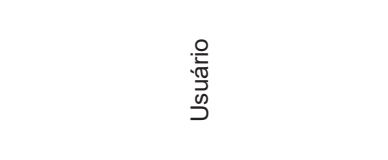 } & 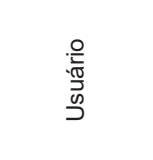 & 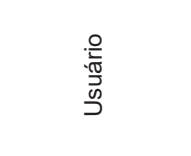 & 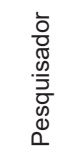 \\
\hline 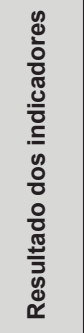 & 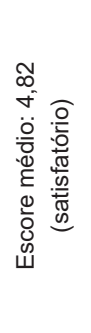 & 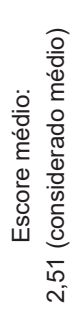 & 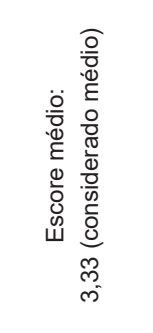 & 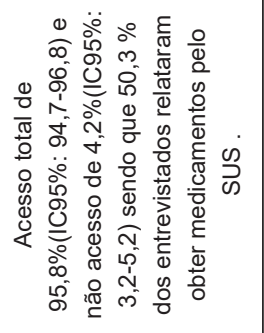 & 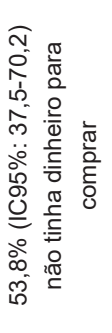 & 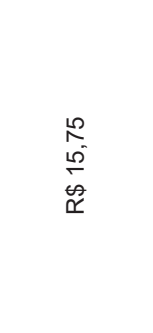 & 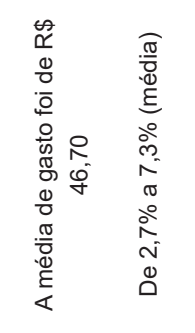 & 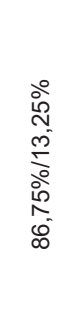 \\
\hline 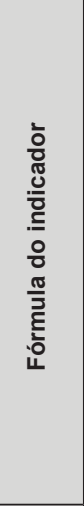 & \multicolumn{3}{|c|}{ 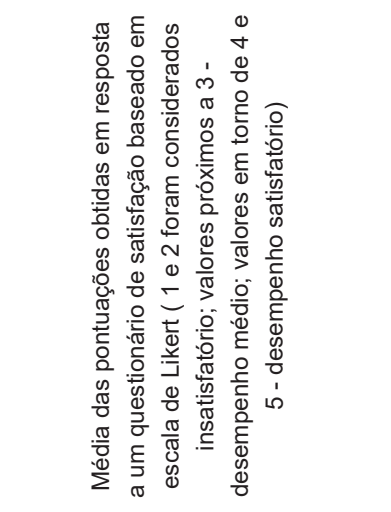 } & 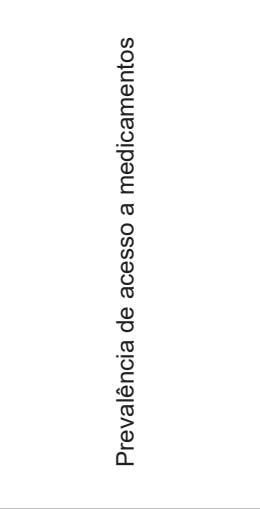 & 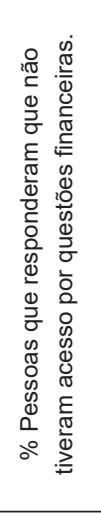 & 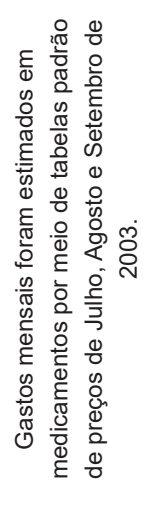 & 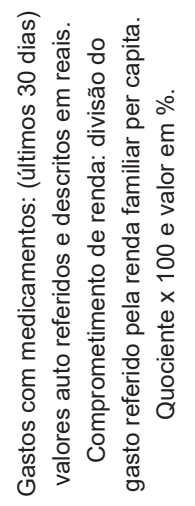 & 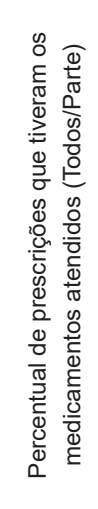 \\
\hline 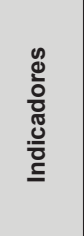 & 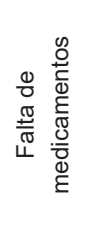 & 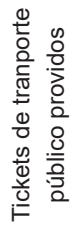 & 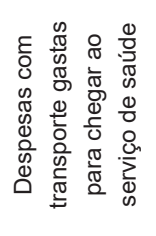 & 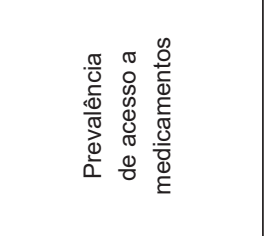 & 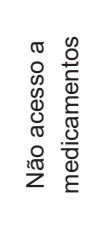 & 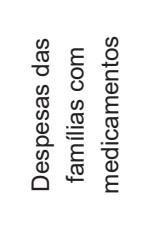 & 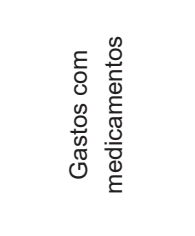 & 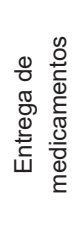 \\
\hline 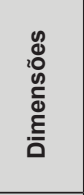 & 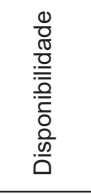 & 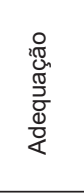 & 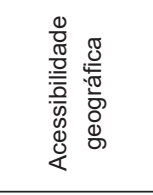 & 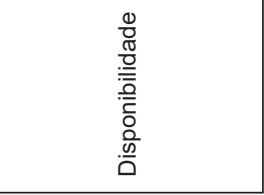 & 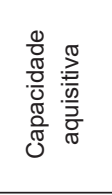 & 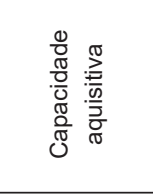 & 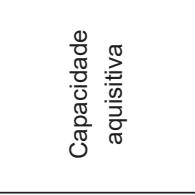 & 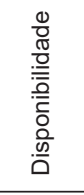 \\
\hline 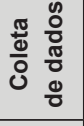 & \multicolumn{3}{|c|}{ 仓े } & \multicolumn{2}{|l|}{ 㝘 울 } & ̊ి & 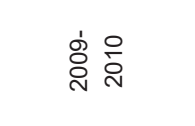 & $\bar{i}$ \\
\hline 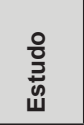 & \multicolumn{3}{|c|}{ 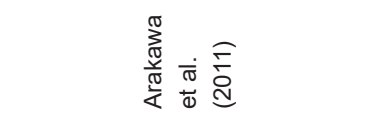 } & \multicolumn{2}{|l|}{ 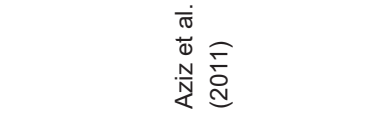 } & 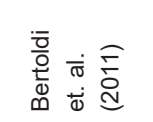 & 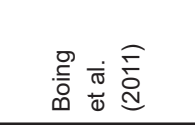 & 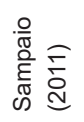 \\
\hline
\end{tabular}




\begin{tabular}{|c|c|c|c|c|c|c|c|c|}
\hline 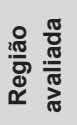 & \multicolumn{2}{|c|}{$\overline{\bar{\omega}}$} & & $\overline{\bar{S}}$ & 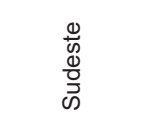 & & $\begin{array}{l}\overline{\bar{\pi}} \\
\frac{\bar{\pi}}{\bar{m}}\end{array}$ & $\begin{array}{l}\overline{\bar{\pi}} \\
\overline{\tilde{D}} \\
\bar{\omega}\end{array}$ \\
\hline 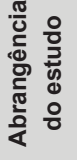 & \multicolumn{2}{|c|}{ 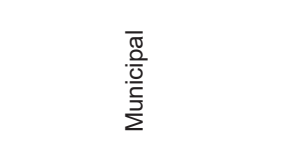 } & \multicolumn{2}{|r|}{ 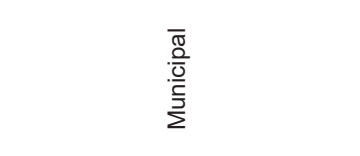 } & $\begin{array}{l}\frac{\overline{0}}{\frac{.0}{\bar{l}}} \\
\frac{\overline{\bar{E}}}{2}\end{array}$ & \multicolumn{2}{|c|}{$\begin{array}{l}\overline{\widetilde{\pi}} \\
\overline{0} \\
\frac{\overline{0}}{\bar{\pi}} \\
z\end{array}$} & $\begin{array}{l}\overline{\widetilde{\sigma}} \\
\overline{\mathrm{O}} \\
\overline{\mathrm{N}} \\
\mathrm{\pi}\end{array}$ \\
\hline 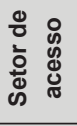 & \multicolumn{2}{|c|}{ 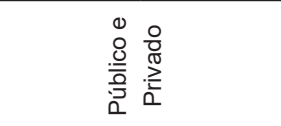 } & \multicolumn{2}{|r|}{ 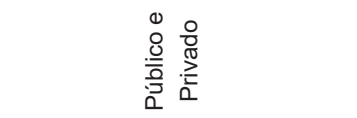 } & $\frac{8}{\frac{8}{9}}$ & \multicolumn{2}{|c|}{ 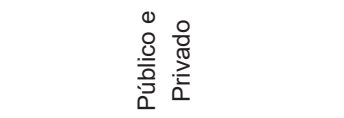 } & 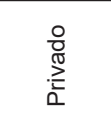 \\
\hline 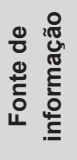 & \multicolumn{2}{|c|}{ 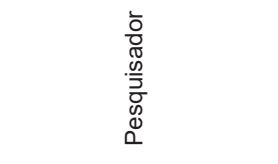 } & \multicolumn{2}{|r|}{ 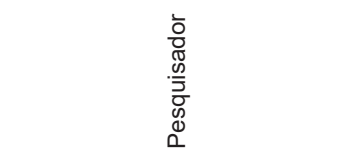 } & 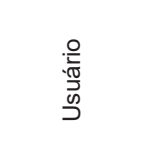 & \multicolumn{2}{|c|}{ 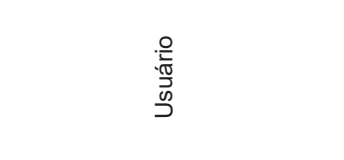 } & 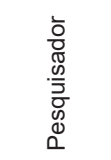 \\
\hline 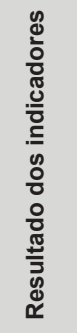 & 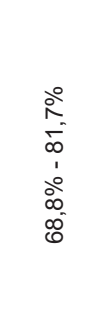 & 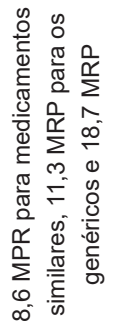 & 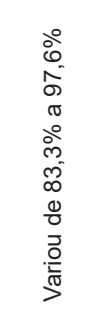 & 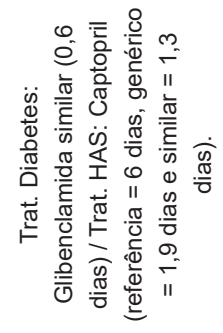 & 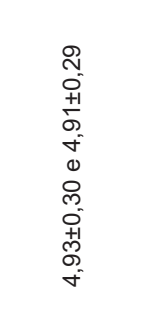 & 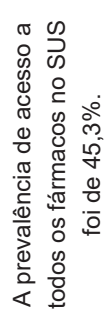 & 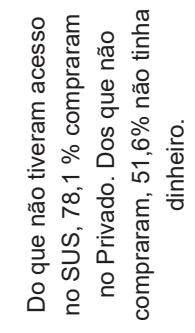 & $\begin{array}{l}\text { o } \\
\text { on } \\
\text { in } \\
\text { of }\end{array}$ \\
\hline 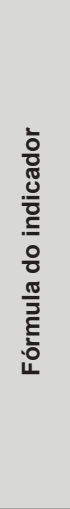 & 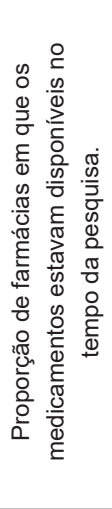 & 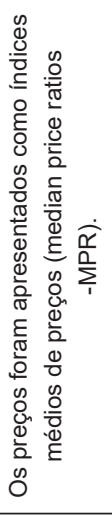 & 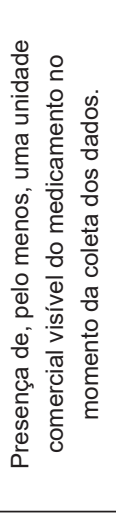 & 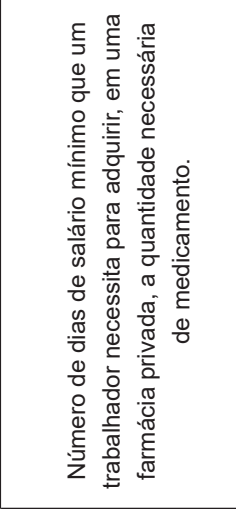 & 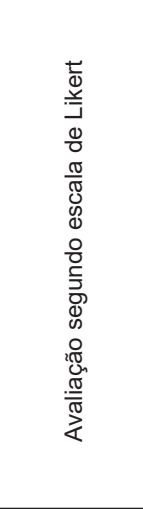 & 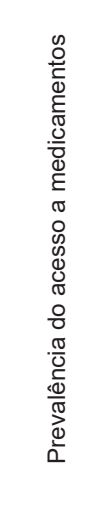 & 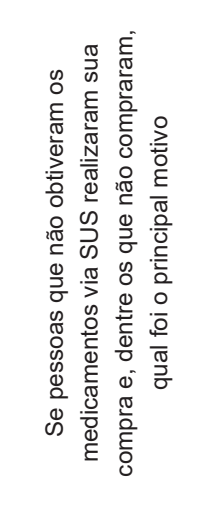 & 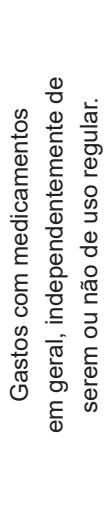 \\
\hline 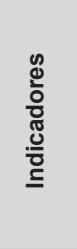 & 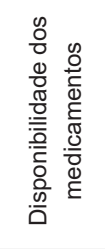 & 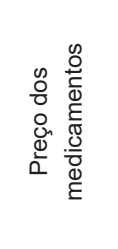 & 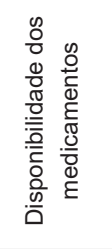 & 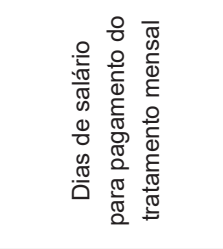 & 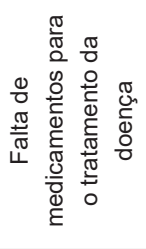 & 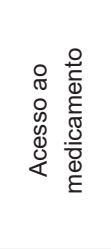 & 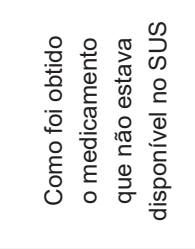 & 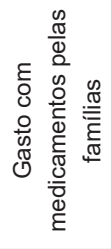 \\
\hline 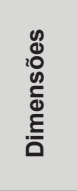 & 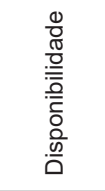 & 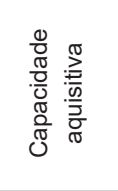 & 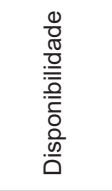 & 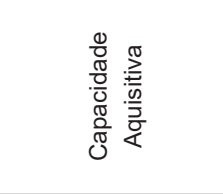 & 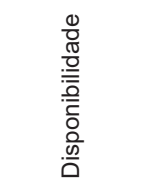 & 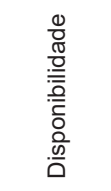 & 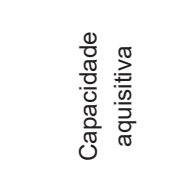 & 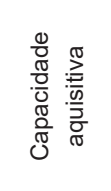 \\
\hline 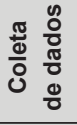 & \multicolumn{2}{|c|}{ ò } & \multicolumn{2}{|r|}{ 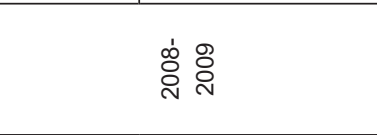 } & 客 完 & \multicolumn{2}{|r|}{$\stackrel{\infty}{\stackrel{\infty}{\sim}}$} & 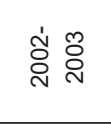 \\
\hline $\begin{array}{l}\text { 을 } \\
\text { 耑 }\end{array}$ & \multicolumn{2}{|c|}{ 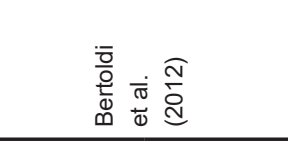 } & & 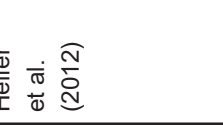 & 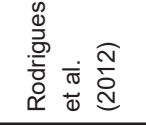 & \multicolumn{2}{|c|}{ 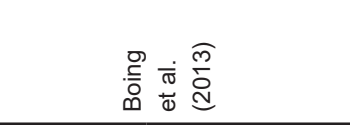 } & 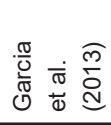 \\
\hline
\end{tabular}




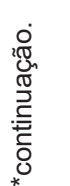

\begin{tabular}{|c|c|c|c|c|c|}
\hline 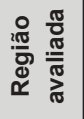 & $\overline{\bar{\omega}}$ & $\begin{array}{l}\overline{\bar{\pi}} \\
\frac{\mathscr{\omega}}{\bar{\omega}}\end{array}$ & \multicolumn{3}{|c|}{$\begin{array}{l}\overline{\bar{\pi}} \\
\overline{\mathscr{\omega}} \\
\overline{\bar{\omega}}\end{array}$} \\
\hline 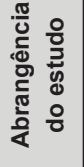 & 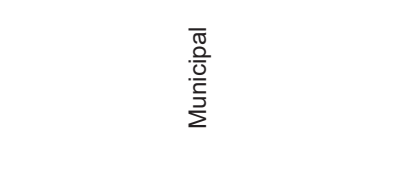 & $\begin{array}{l}\overline{\widetilde{\sigma}} \\
\stackrel{0}{0} \\
\overline{0} \\
\bar{\pi}\end{array}$ & \multicolumn{3}{|c|}{$\begin{array}{l}\bar{\sigma} \\
. \overline{0} \\
\frac{0}{\bar{\pi}} \\
z\end{array}$} \\
\hline 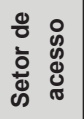 & 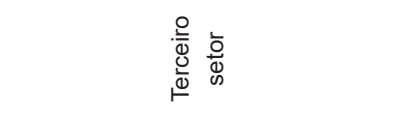 & $\begin{array}{l}0 \\
\stackrel{8}{0} \\
\stackrel{2}{2} \\
0\end{array}$ & \multicolumn{3}{|c|}{$\frac{8}{\overline{0}}$} \\
\hline 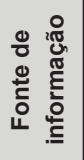 & 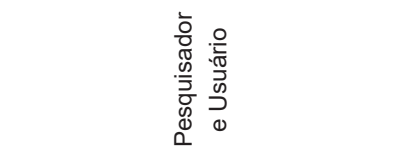 & 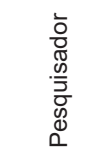 & \multicolumn{3}{|c|}{ 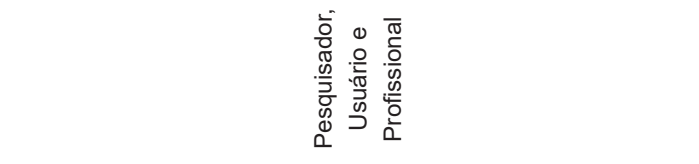 } \\
\hline 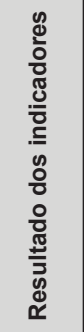 & 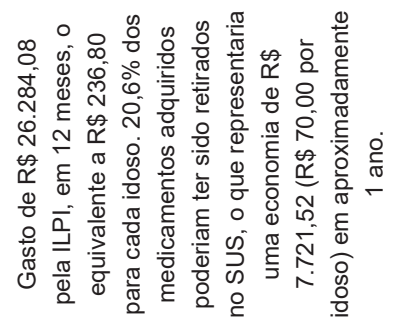 & 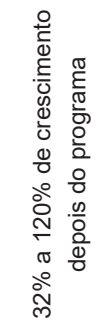 & 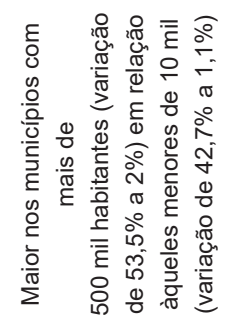 & 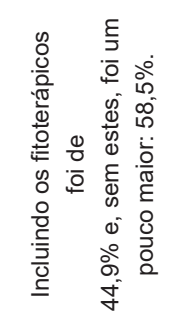 & 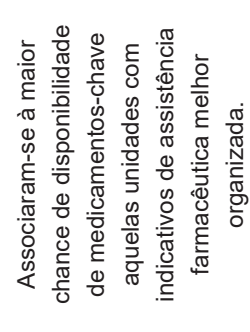 \\
\hline 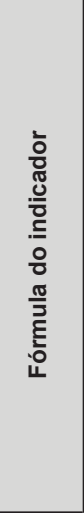 & 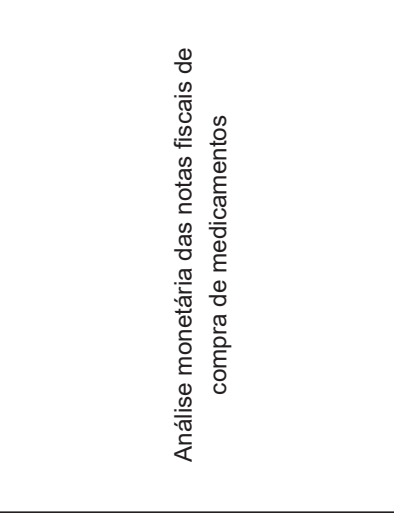 & 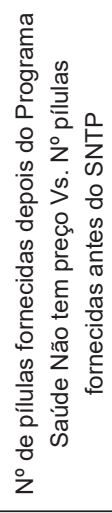 & \multicolumn{3}{|c|}{ 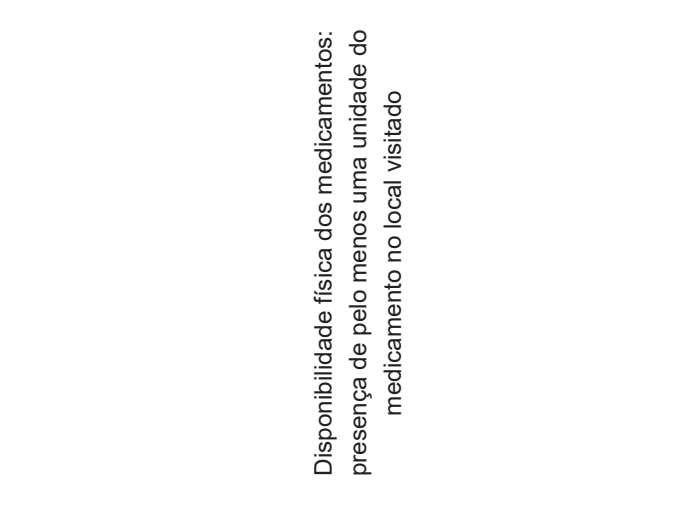 } \\
\hline 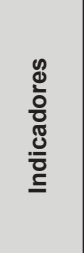 & 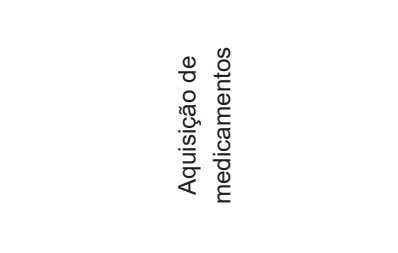 & 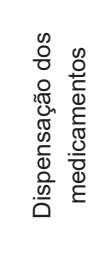 & 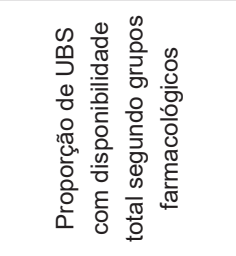 & 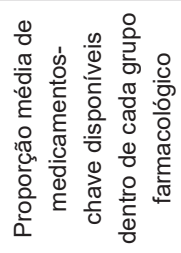 & 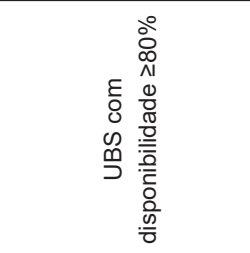 \\
\hline 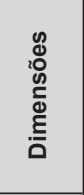 & 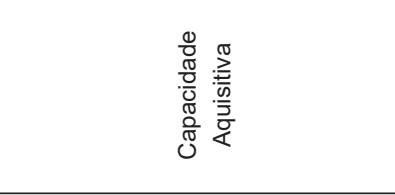 & 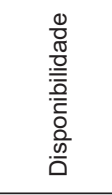 & \multicolumn{3}{|c|}{ 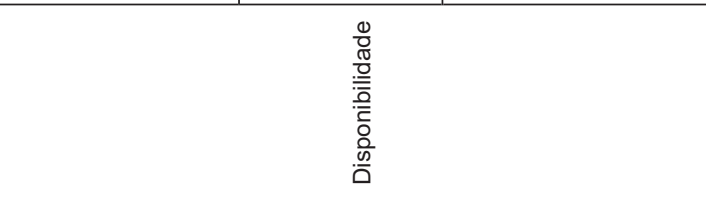 } \\
\hline 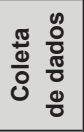 & 㝘 울 & 홀 & \multicolumn{3}{|c|}{ 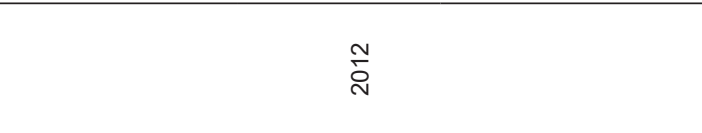 } \\
\hline 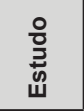 & 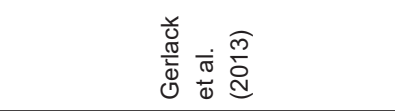 & 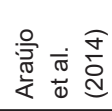 & \multicolumn{3}{|c|}{ 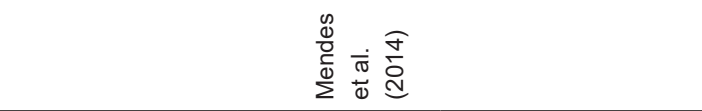 } \\
\hline
\end{tabular}

0
20
0
0
0
0
0
$*$
$*$ 
O acesso a medicamentos essenciais (segundo relações padronizadas ou classificação da OMS) foi avaliado em 22 estudos $(76 \%)$ e os outros sete estudos avaliaram tipos específicos de medicamentos (psicofármacos, medicamentos para tuberculose, HIV, Alzheimer) ou não relataram o tipo de medicamento avaliado.

A classificação dos indicadores segundo as dimensões de acesso revelou que dos 29 estudos incluídos, 10 (34\%) avaliaram apenas a dimensão "Disponibilidade" e cinco (17\%) avaliaram "Capacidade Aquisitiva". Os outros 14 estudos avaliaram mais de uma dimensão do acesso: dez estudos, "Disponibilidade e Capacidade Aquisitiva", um, "Disponibilidade e Acomodação", e dois estudos avaliaram três dimensões do acesso, sendo um "Disponibilidade, Acomodação e Acessibilidade Geográfica" e um, "Disponibilidade, Aceitabilidade e Capacidade Aquisitiva". No total, 24 estudos (83\%) apresentaram indicadores de

Tabela 2 - Indicadores utilizados nos estudos incluidos, classificados de acordo com as dimensões de acesso propostas por Penchansky e Thomas (1981)

\begin{tabular}{|c|c|c|c|}
\hline $\begin{array}{l}\text { Dimensões do } \\
\text { Acesso }\end{array}$ & Indicadores utilizados para medir acesso & $\mathrm{N}^{\circ}$ estudos & Resultados \\
\hline \multirow{5}{*}{ Disponibilidade } & $\begin{array}{l}\text { Disponibilidade física dos medicamentos: presença de pelo } \\
\text { menos uma unidade do medicamento no local visitado }\end{array}$ & 12 estudos & $30 \%-90,2 \%$ \\
\hline & $\begin{array}{c}\text { - Não acesso e o tempo de desabastecimento: quantidade } \\
\text { de medicamentos que não estavam disponíveis (Escala de } \\
\text { Likert)* }\end{array}$ & 2 estudos & Escore médio: 4,9 \\
\hline & $\begin{array}{c}\text { - Percentual de prescrições que tiveram os medicamentos } \\
\text { atendidos; }\end{array}$ & 3 estudos & $54 \%-87 \%$ \\
\hline & $\cdot$ Ter acesso ao medicamento; & 6 estudos & $25 \%-87 \%$ \\
\hline & $\begin{array}{l}\text { Percepção da Disponibilidade de serviços farmacêuticos } \\
\text { (Escala de Likert })^{\star *}\end{array}$ & 1 estudo & Escore variou de 13 a $33^{* *}$ \\
\hline \multirow{3}{*}{$\begin{array}{l}\text { Acessibilidade } \\
\text { Geográfica }\end{array}$} & $\begin{array}{c}\text { - Avaliação das despesas com transporte, gastas para chegar } \\
\text { ao serviço de saúde (Escala de Likert)* }\end{array}$ & 1 estudo & Escore médio: 3,33 \\
\hline & & & \\
\hline & · Distância geográfica das UBS & 1 estudo & $\begin{array}{l}48,7 \% \text { são da área de } \\
\text { abrangência e } 51,3 \% \text { são } \\
\text { usuários de outras áreas }\end{array}$ \\
\hline \multirow{5}{*}{ Adequação } & $\begin{array}{l}\text { - Avaliação do provimento de tickets de transporte gratuito } \\
\text { (Escala de Likert)* }\end{array}$ & 1 estudo & Escore médio: 2,51 \\
\hline & $\cdot$ Tempo médio de consulta & 1 estudo & 14 minutos \\
\hline & $\begin{array}{c}\text { - Disponibilidade de cópias da RME nas salas de } \\
\text { atendimento }\end{array}$ & 1 estudo & Não foi possível observar \\
\hline & $\cdot$ Tempo médio de dispensação & 1 estudo & 3 minutos \\
\hline & - Percentagem dos pacientes que sabiam a posologia & 1 estudo & $70 \%$ \\
\hline \multirow{6}{*}{$\begin{array}{l}\text { Capacidade } \\
\text { Aquisitiva }\end{array}$} & $\begin{array}{l}\text { - Custo ou preço do medicamento para o paciente (Índice } \\
\text { médio de preços) }\end{array}$ & 7 estudos & $\begin{array}{l}\text { O índice médio de preços } \\
\text { (Median Price Ratio-MPR) } \\
\text { variou de } 2,02 \text { a } 18,7\end{array}$ \\
\hline & $\begin{array}{l}\text { Gasto necessário para o tratamento relacionado ao número } \\
\text { de dias trabalhados no mês em função ao salário mínimo }\end{array}$ & 5 estudos & 0,25 a 13,7 dias \\
\hline & - Indicadores de gasto bruto com medicamentos & 1 estudo & $\begin{array}{c}\mathrm{R} \$ 26.284,08 \text { pela ILPI, em } 12 \\
\text { meses }\end{array}$ \\
\hline & - Gasto médio mensal com medicamentos pelas famílias & 3 estudos & $\mathrm{R} \$ 15,75$ a $\mathrm{R} \$ 59,02$ \\
\hline & $\begin{array}{c}\text { - Maneira de obtenção de medicamentos não disponíveis } \\
\text { pelo SUS }\end{array}$ & 1 estudo & $\begin{array}{l}78,1 \% \text { compraram no Privado. } \\
51,6 \% \text { dos que não compraram, } \\
\text { o fizeram por falta de dinheiro. }\end{array}$ \\
\hline & · Percepção da capacidade aquisitiva & 1 estudo & Escore variou de 2 a $5^{* *}$ \\
\hline Aceitabilidade & $\begin{array}{l}\text { · Percepção da satisfação com a última visita à farmácial } \\
\text { Unidade de dispensação. }\end{array}$ & 1 estudo & Escore variou de 18 a $51^{* *}$ \\
\hline
\end{tabular}


"Disponibilidade" e 11 estudos, indicadores de "Capacidade Aquisitiva". Nenhum estudo avaliou todas as dimensões do acesso segundo a classificação de Penchansky e Thomas ${ }^{9}$ (Quadro 1).

Quanto aos indicadores utilizados para medir o acesso, classificados de acordo com as dimensões propostas por Penchansky e Thomas", foram encontrados 19 indicadores diferentes. $\mathrm{O}$ indicador "disponibilidade física do medicamento" foi o mais avaliado nos estudos. Os resultados dos indicadores apresentaram grande variabilidade, por exemplo, a disponibilidade física do medicamento variou de $30-90,2 \%$ e o índice médio de preços de 2,02-18,7 (Tabela 2).

Com relação à avaliação da qualidade dos estudos incluídos, vários itens da ferramenta foram classificados como "Não se aplica", uma vez que o delineamento dos estudos foi na quase totalidade transversal descritivo, sem grupo controle. Entretanto, a avaliação da qualidade pôde ser considerada como adequada, uma vez que o percentual consolidado de respostas favoráveis foi superior a $80 \%$ para todas as questões avaliadas.

\section{DISCUSSÃO|}

Os estudos incluídos nesta revisão sistemática foram classificados de acordo com as dimensões de acesso descritas por Penchansky e Thomas ${ }^{9}$ a fim de sistematizar os dados e avaliar se existe um padrão de medidas nos estudos realizados no Brasil.

De uma maneira geral, a maioria dos estudos utilizou indicadores de Disponibilidade, sendo relativos a "tempo de desabastecimento", "percentual de um conjunto de elementos essenciais disponíveis em estoque", percentual ou prevalência de acesso e prescrições atendidas.

A dimensão do "Capacidade Aquisitiva" foi a segunda mais avaliada nos estudos incluídos e teve como principal indicador o custo ou preço do medicamento para o paciente (Índice médio de preços).

Aceitabilidade foi avaliada em apenas um estudo ${ }^{10}$, que mediu a percepção da satisfação do usuário com os serviços da farmácia. A relação direta com preferências e interpretações dos usuários acerca de outras dimensões do acesso dificulta a elaboração e compreensão das medidas. Logo, uma abordagem qualitativa e a aplicação de questionários de satisfação podem ser alternativas consideráveis para a efetivação destas medidas.

Acessibilidade geográfica foi avaliada em dois estudos ${ }^{21,35} \mathrm{e}$ apresentou como um dos indicadores a "distância geográfica da UBS até o domicílio do usuário"; entretanto, não foi utilizada uma distância padronizada. Já a Organização Mundial de Saúde descreve como indicador o percentual de domicílios situados a mais de cinco quilômetros de um centro de saúde/farmácia. A organização por população adscrita, ou seja, da população da área de abrangência de uma unidade de saúde, adotada na atenção básica provavelmente minimiza os problemas de "Acessibilidade Geográfica"; entretanto suas medidas se fazem necessárias, em especial nas regiões de difícil acesso e mais distantes do país e no que tange aos serviços mais especializados.

Para a dimensão "Acomodação", um estudo ${ }^{38}$ apresentou vários indicadores, como "tempo médio de consulta e dispensação", "percentual de inscritos em um programa de tratamento que realmente estavam ativos" e a "disponibilidade de cópias da Relação de Medicamentos Essenciais e RENAME nas salas de atendimento". Os indicadores apresentados neste artigo se aproximam dos indicadores da OMS "existência de uma lista de medicamentos essenciais" e "percentual de instalações que têm uma fonte de informação sobre os medicamentos, incluindo receituário". Os indicadores de "Acomodação" aparecem com frequência reduzida nos estudos, o que pode ser devido a uma maior associação da avaliação da "Acomodação" com o cumprimento de critérios legais do que como uma dimensão do acesso em si.

Dos 29 estudos incluídos, mais da metade baseou-se em relatos dos usuários. Entretanto, é importante ressaltar que estudos feitos exclusivamente com usuários são passíveis de viés de memória, visto que a avaliação do usuário dá maior peso ao último atendimento recebido em detrimento dos outros. Uma forma de tentar minimizar este fato é a elaboração de estudos que coletem dados de mais de uma fonte, com o fim de compará-los. Um exemplo simples seria a coleta de dados dos profissionais de saúde e dos usuários do serviço, entretanto, somente quatro estudos utilizaram fontes mistas para a coleta de dados. Este é um dado de destaque, uma vez que o acesso a medicamentos envolve vários atores, constituindo um fluxo indissociável que vai desde a pesquisa e a produção até o consumo, passando 
por instâncias políticas, gestoras e produtivas. Por isso, quanto maior o número de atores sociais envolvidos na avaliação do acesso, mais completos os resultados oriundos desses trabalhos.

Dado que a maioria dos estudos objetivou a avaliação de medicamentos essenciais, tem-se um indicativo de que poucos estudos avaliaram medicamentos de maior valor agregado, como medicamentos do componente especializado, por exemplo, de disponibilização mais restrita. Contrapondo-se em relação à frequência da elaboração deste tipo de estudo, curiosamente, os artigos sobre judicialização se multiplicam a cada dia, o que pode indicar uma lacuna de acesso para este tipo de produto.

O fato de que sete dos 29 artigos encontrados abordam de alguma forma a região nordeste do país e nenhum aborda exclusivamente a região norte, pode ser indício de um viés regional na mensuração do acesso.

No setor público, avaliou-se mais a "Disponibilidade" do que as outras dimensões, possivelmente pelo fato de que uma avaliação da "Capacidade Aquisitiva" do usuário não faz muito sentido, uma vez que os medicamentos são disponibilizados gratuitamente no SUS. No entanto, alguns estudos realizaram a análise do impacto da compra dos medicamentos não-obtidos no SUS sobre a renda das famílias, indicador interessante na compreensão global do acesso. No setor privado, a "Capacidade Aquisitiva" teve maior espaço, já que é possível fazer uma análise de preços de venda e do comprometimento da renda familiar com medicamentos, por exemplo. A "Disponibilidade" nas farmácias comerciais é passível de mensuração e usualmente é alta, dado o caráter comercial destas unidades.

Um ponto pouco abordado foi a adequação do serviço às necessidades dos usuários. Os estudos encontrados revelaram que os usuários parecem aceitar bem as condições de atendimento nas unidades. No entanto, o funcionamento das unidades de saúde em horário comercial é um fator limitante na promoção da saúde e acesso a medicamentos para a população economicamente ativa. Desta forma, o acesso de grande parte da população inserida no mercado de trabalho se faz somente em situações de urgência e emergência, fato que não foi captado nos estudos.

Foi observado um foco na mensuração de Disponibilidade física e indicadores relativos a gastos e preços, sem a avaliação completa do acesso, como processo multifacetado, envolvendo também usuários e a estruturação do sistema. Isso pode estar relacionado à relativa simplicidade da obtenção desses dados, mediante entrevistas ou mesmo consulta a listagem de preços-padrão.

O acesso a medicamentos como parte integrante do acesso aos serviços de saúde é um conceito multidimensional, ou seja, é representado por um conjunto de características que vão muito além da simples disponibilização física do produto farmacêutico, pois o usuário que recorre ao medicamento o faz visando, acima de tudo, o símbolo de saúde que ele representa, buscando não somente um alívio (quase mágico) para a sintomatologia de seu agravo à saúde, mas também para satisfazer uma série de outras expectativas, como desejo de bem estar, de proteção, de performance, entre outros ${ }^{50}$. Tal fato pode estar relacionado com o próprio modelo de cuidado à saúde vigente, centrado mais nas tecnologias em saúde (incluindo os medicamentos) do que no paciente e nas suas preferências. Logo, a forma como é mensurado o acesso a medicamentos pode refletir na filosofia do cuidado, que prioriza a oferta dos produtos e não a forma como estes são ofertados.

O acesso a medicamentos no Brasil ainda é um grande desafio, uma vez que os indicadores revelaram resultados bem variáveis, que foram desde uma escassez preocupante de medicamentos até um suprimento satisfatório acima de $90 \%$. Em um mesmo município foram encontrados diferentes níveis de acesso a medicamentos. Essa variabilidade sugere que a gestão local dos serviços de atenção básica tem papel preponderante na efetivação do acesso a medicamentos.

A avaliação do acesso não tem sido alvo de averiguações sistemáticas. A ausência de uma padronização dos indicadores e a heterogeneidade das medidas de acesso foi um fator limitante, inviabilizando uma meta-síntese dos dados, a qual poderia subsidiar a elaboração de um panorama mais completo do acesso no Brasil.

\section{CONCLUSÃO |}

A maioria dos estudos de acesso a medicamentos no Brasil é do setor público utiliza como fonte de dados os usuários e avalia medicamentos essenciais. Avaliou-se primariamente a disponibilidade e nenhum estudo avaliou todas as dimensões do acesso a medicamentos. O nível de 
acesso mostrou grande variabilidade entre os estudos, com ausência de sistematização nas medidas e indicadores de avaliação do acesso.

Esta avaliação levanta a necessidade de desenvolver uma diretriz que proponha uma padronização de medidas para avaliar acesso a medicamentos, que fomentem comparações e avaliações do desempenho dos sistemas de saúde ao longo do tempo.

\section{REFERÊNCIAS |}

1. Hogerzeil HV, Mirza Z. The world medicines situation 2011: access to essential medicines as part of the right to health [Internet]. Geneva: World Health Organization; 2011 [acesso em 04 nov 2014]. Disponível em: URL: <http:// apps.who.int/medicinedocs/documents/s18772en/ s18772en.pdf $>$.

2. Programa das Nações Unidas Para o Desenvolvimento [nternet]. Objetivos de desenvolvimento do milênio [acesso em 04 nov 2014]. Disponível em: URL: <http:// www.pnud.org.br/odm>.

3. World Health Organization [Internet]. Primary health care: report of the International Conference on Primary Health Care, Alma Ata** [acesso em 04 nov 2014]. Geneva: World Health Organization; 1978. Disponível em: URL: $<$ http://www.searo.who.int/entity/primary_health_care/ documents/hfa_s_1.pdf $>$.

4. World Health Organization [Internet]. Report of the WHO expert committee on national drug policies: contribution to updating the WHO guidelines for developing national drug policies [acesso em 04 nov 2014] Geneva: World Health Organization; 1995. Disponível em: URL: <http://apps.who.int/medicinedocs/documents/ s16221e/s16221e.pdf $>$.

5. Brasil. Constituição (1988) [Internet]. Constituição da República Federativa do Brasil [acesso em 30 set 2014]. Brasília, DF: Senado Federal; 1988. Disponível em: URL: <http://www.planalto.gov.br/ccivil_03/constituicao/ constituicao.htm $>$.

6. Brasil. Lei $\mathrm{n}^{\circ}$. 8.080, de 19 de setembro de 1990 . Dispõe sobre as condições para a promoção, proteção e recuperação da saúde, a organização e o funcionamento dos serviços correspondentes e dá outras providências [Internet]. Diário Oficial da União 20 set 1990 [acesso em 30 set 2014];Seção 1. Disponível em: URL: <http://www. planalto.gov.br/ccivil_03/Leis/L8080.htm>.

7. World Health Organization [Internet]. How to develop and implement a national drug policy. 2. ed. Geneva: World Health Organization; 1988 [acesso em 04 nov 2014]. Disponível em: URL: <http://whqlibdoc.who.int/ publications $/ 924154547 \mathrm{X} . \mathrm{pdf}>$.

8. Luiza VL. Acesso a medicamentos essenciais no estado do Rio de Janeiro. Rio de Janeiro. Tese [Doutorado em Saúde Pública] - Escola Nacional De Saúde Pública; 2003.

9. Penchansky R, Thomas JW. The concept of access: definition and relationship to consumer satisfaction. Med Care. 1981; 19(2):127-140.

10. Emmerick ICM. Dimensões e determinantes do acesso a medicamentos em três países da América Central. Rio de Janeiro. Tese [Doutorado em Saúde Pública] - Escola Nacional de Saúde Pública Sergio Arouca; 2011.

11. Instituto Brasileiro de Geografia e Estatística. Síntese de indicadores sociais: uma análise das condições de vida da população brasileira. Rio de Janeiro; 2013.

12. Costa KS, Nascimento-Júnior JM, Soeiro OM, Paganelli MO, Araújo SQ. Assistência farmacêutica nas redes de atenção à saúde do SUS. In: Brasil. Ministério da Saúde. Cuidado farmacêutico na atenção básica: serviços farmacêuticos na atenção básica à saúde. Brasília: Ministério da Saúde; 2014. p. 25-36.

13. Lage A. Global pharmaceutical development and access: critical issues of ethics and equity. MEDICC Review. 2011; 13(3):16-22

14. Brasil. Ministério da Saúde. Secretaria de Vigilância em Saúde. Programa nacional de imunizações: 30 anos. Brasília, DF: Ministério da Saúde; 2003.

15. Brasil. Ministério da Saúde. Secretaria-Executiva. Ministério da Saúde e municípios: juntos pelo acesso integral e de qualidade à saúde. 2. ed. Brasília, DF: 2013.

16. Marin N, Luiza VL, Castro CGSO, Santos SM. 
Assistência farmacêutica para gerentes municipais. Rio de Janeiro: OPAS/OMS; 2003.

17. Karnikowski MGO, Nóbrega OT, Naves JOS \& Silver LD. Access to essential drugs in 11 Brazilian cities: a community based evaluation and action method. J Public Health Policy. 2004; 25(3/4):288-98.

18. Oliveira LCF, Assis MMA, Barboni AR. Assistência farmacêutica no Sistema Único de Saúde: da política nacional de medicamentos à atenção básica à saúde. Ciênc Saúde Coletiva. 2010; 15(3):3561-7.

19. Boing AC, Bertoldi AD, Boing AF, Bastos JL, Peres KG. Acesso a medicamentos no setor público: análise de usuários do Sistema Único de Saúde no Brasil. Cad Saúde Pública. 2013; 29(4):691-701.

20. Oliveira BB. Investigações de acesso a medicamentos em nível domiciliar: um artigo comparativo de iniciativas realizadas no Brasil entre 2002 e 2004. Rio de Janeiro. Tese [Mestrado em Saúde Pública] - Escola Nacional de Saúde Pública; 2006.

21. Arakawa T, Arcêncio RA, Scatolin BE, Scatena LM, Ruffino-Netto A, Villa TCS. Accessibility to tuberculosis treatment: assessment of health service performance. Rev Lat Am Enfermagem. 2011; 19(4):994-1002.

22. Araujo JLO, Pereira MD, Fiol FSD, Barberato-Filho S. Access to Antihypertensive agents in Brazil: evaluation of the "Health Has No Price" program. Clin Ther. 2014; 36(8):1191-5.

23. Bertoldi AD, Helfer AP, Camargo AL, Tavares NUL, Karanavos P. Medicine prices, availability and affordability in Southern Brazil: a study of public and private facilities. London: LSE Health; 2010.

24. Bertoldi AD, Helfer AP, Camargo AL, Tavares NUL, Kanavos P. Is the Brazilian pharmaceutical policy ensuring population access to essential medicines? Global Health. 2012; 8:6.

25. Ferreira-Filho JCR, Correia GT, Mastroianni PC. Acesso a medicamentos essenciais em farmácias e drogarias do Município de Araraquara. Rev Ciênc Farm Básica Apl. 2010; 31(2):177-82.
26. Sampaio GC. Análise do acesso a medicamentos em uma unidade do programa de saúde da família em Porto Alegre. Rio Grande do Sul. Monografia [Graduação em Farmácia] - Universidade Federal do Rio Grande do Sul; 2011.

27. Gerlack LF, Bós AJG, Lyra-Junior DP, Karnikowski MG. O acesso e aquisição de medicamentos em instituição de longa permanência para idosos no Brasil. Sci Med (Porto Alegre). 2013; 23(2):90-5.

28. Guerra-Junior AA, Acurcio FA, Gomes GAP, Miralles M, Girardi SN, Werneck GAF, et al. Disponibilidade de medicamentos essenciais em duas regiões de Minas Gerais, Brasil. Rev Panam Salud Publica. 2004; 15(3):168-75.

29. Helfer AP, Camargo AL, Tavares NUL, Kanavos P, Bertoldi AD. Capacidade aquisitiva e disponibilidade de medicamentos para doenças crônicas no setor público. Rev Panam Salud Publica. 2012; 31(3):225-32.

30. Mendis S, Fukino K, Cameron A, Laing R, Filipe-Junior A, Khatib O, et al. The availability and affordability of selected essential medicines for chronic diseases in six lowand middle-income countries. Bull World Health Organ. 2007; 85(4):279-88.

31. Miranda ES, Pinto CDBS, Reis ALA, Emmerick ICM, Campos MR, Luiza VL, et al. Disponibilidade no setor público e preços no setor privado: um perfil de medicamentos genéricos em diferentes regiões do Brasil. Cad Saúde Pública. 2009; 25(10):2147-58.

32. Paniz VMV, Fassa ACG, Facchini LA, Piccini RX, Tomasi E, Thumé E, et al. Free access to hypertension and diabetes medicines among the elderly: a reality yet to be constructed. Cad Saúde Pública. 2010; 26(6):1163-74.

33. Paniz VMV, Fassa ACG, Facchini LA, Bertoldi AD, Piccini RX, Tomasi E, et al. Acesso a medicamentos de uso contínuo em adultos e idosos nas regiões Sul e Nordeste do Brasil. Cad Saúde Pública. 2008; 24(2):267-80.

34. Rodrigues AMS, Scatena LM, Vendramini SHF, Canini SRMS, Villa TCS, Gir El. Avaliação do acesso ao tratamento de tuberculose por coinfectados ou não pelo vírus da imunodeficiência humana. Rev Esc Enferm USP. 2012; 46(5):1163-9. 
35. Tasca RS, Soares DA, Cuman RKN. Acesso a medicamentos anti-hipertensivos em unidade básica de saúde em Maringá - Paraná. Arq Ciênc Saúde Unipar. 1999; $3(2): 117-24$.

36. Torreao NKAM. Relação Nacional de Medicamentos Essenciais (RENAME) e sua influência no acesso aos medicamentos. São Paulo. Dissertação [Mestrado em Saúde Pública] - Universidade de São Paulo; 2005.

37. Aziz MM, Calvo MCC, Schneider IJC, Xavier AJ, D'orsi E. Prevalência e fatores associados ao acesso a medicamentos pela população idosa em uma capital do sul do Brasil: um artigo de base populacional. Cad Saúde Pública. 2011; 27(10):1939-50.

38. Chaves GC, Emmerick I, Pouvourville N, Saint-Denis T, Fonseca ASA, Luiza VL. Indicadores de uso racional de medicamentos e acesso a medicamentos: um artigo de caso. Rev Bras Farm. 2005; 86(3):97-103.

39. Brasil. Ministério da Saúde. Organização PanAmericana da Saúde. Avaliação da assistência farmacêutica no Brasil. Brasília, DF: Ministério da Saúde; 2005.

40. Garcia LP, Sant'Anna AC, Magalhães LCG, Freitas LRS, Aurea AP. Gastos das famílias brasileiras com medicamentos segundo a renda familiar: análise da Pesquisa de Orçamentos Familiares de 2002-2003 e de 2008-2009. Cad Saúde Pública. 2013; 29(8):1605-16.

41. Bertoldi AD, Barros AJ, Camargo AL, Hallal PC, Vandoros S, Wagner A, et al. Household expenditures for medicines and the role of free medicines in the Brazil public health system. Am J Public Health. 2011; 101(5):916-21.

42. Boing AC, Bertoldi AD, Peres LG. Desigualdades socioeconômicas nos gastos e comprometimento da renda com medicamentos no Sul do Brasil. Rev Saúde Pública. 2011; 45(5):897-905.

43. Boing AC, Bertoldi AD, Boing AF, Bastos JL, Peres KG. Acesso a medicamentos no setor público: análise de usuários do Sistema Único de Saúde no Brasil. Cad Saúde Pública. 2013; 29(4):691-701.

44. Suh GH, Wimo A, Gauthier S, O’Connor D, Ikeda $\mathrm{M}$, Homma A, et al. International price comparisons of
Alzheimer's drugs: a way to close the affordability gap. Int Psychogeriatr. 2009; 21(6):1116-26.

45. Monteiro WM, Melo GC, Massunari GK, Hübner DV, Tasca RS. Avaliação da disponibilidade de medicamentos genéricos em farmácias e drogarias de Maringá (PR) e comparação de seus preços com os de referência e similares. Rev Bras Ciênc Farm. 2005; 41(3):333-43.

46. Pinto CDBS, Miranda ES, Emmerick ICM, Costa NR, Castro CGSO. Preços e disponibilidade de medicamentos no Programa Farmácia Popular do Brasil. Rev Saúde Pública. 2010; 44(4):611-9.

47. Bertoldi AD, Barros AJD, Wagner A, Ross-Degnand D, Hallal PC. Medicine access and utilization in a population covered by primary health care in Brazil. Health Policy. 2009; 89(3):295-302.

48. Miralles MA, Kimberlin CL. Perceived access to care and medication use among ambulatory elderly in Rio de Janeiro, Brazil. Soc Sci Med. 1998; 46(3):345-55.

49. Mendes LV, Campos MR, Chaves GC, Silva RM, Feitas PS, Costa KS, et al. Disponibilidade de medicamentos nas unidades básicas de saúde e fatores relacionados: uma abordagem transversal. Saúde Debate. 2014; 38(n. esp):109-23.

50. Lefèvre F. Saúde, este obscuro objeto de desejo. Saúde Soc. $1997 ; 6(1): 3-9$.

Correspondência para/Reprint request to:

Vânia Eloisa de Araújo

Av. Pres. Antônio Carlos, 6627,

Lagoinha/BH, Brasil

CEP: 31270-901

Tel: (31) 3409-6837

E-mail:vaniaearanjo@gmail.com

Submetido em: 23/07/2015

Aceito em: 21/12/2015 